\title{
Theory of azimuthally small-scale hydromagnetic waves in the axisymmetric magnetosphere with finite plasma pressure
}

\author{
D. Y. Klimushkin \\ Institute of Solar-Terrestrial Physics, Irkutsk 33, P.O.Box 4026, 664033, Russia \\ e-mail: klimush@iszf.irk.ru
}

Received: 3 September 1996 / Revised: 30 April 1997 / Accepted: 6 May 1997

\begin{abstract}
The structure of monochromatic MHD-waves with large azimuthal wave number $m \gg 1$ in a twodimensional model of the magnetosphere has been investigated. A joint action of the field line curvature, finite plasma pressure, and transversal equilibrium current leads to the phenomenon that waves, standing along the field lines, are travelling across the magnetic shells. The wave propagation region, the transparency region, is bounded by the poloidal magnetic surface on one side and by the resonance surface on the other. In their meaning these surfaces correspond to the usual and singular turning points in the WKB-approximation, respectively. The wave is excited near the poloidal surface and propagates toward the resonance surface where it is totally absorbed due to the ionospheric dissipation. There are two transparency regions in a finite-beta magnetosphere, one of them corresponds to the Alfvén mode and the other to the slow magnetosound mode.
\end{abstract}

Key words. Magnetosphere Azimuthally small-scale waves $\cdot$ MHD waves

\section{Introduction}

A significant proportion of the geomagnetic pulsations observed on the ground and in space is made up of waves with a large value of the azimuthal wave number $(m \gg 1)$, henceforth referred to as the azimuthally smallscale waves. Usually among them are the familiar poloidal Pc 4 pulsations and compressional, storm-time Pc 5 pulsations (Anderson, 1994), as well as less common giant Pg pulsations (Takahashi et al., 1992) and hydromagnetic vortices (Saunders et al., 1981). These types of oscillations are all frequently observed in outer magnetospheric regions where plasma is relatively hot: the value of the parameter $\beta$ can be about unity (Zhu and Kivelson, 1991).
Among three hydromagnetic modes in the $m \gg 1$ case, only Alfvén and slow magnetosound (SMS) modes are important in the magnetosphere since fast magnetic sound (FMS) is concentrated near the magnetopause and can not penetrate deep into the magnetosphere (Walker, 1987; Leonovich and Mazur, 1990). Recall that there is no SMS in a cold plasma.

This paper is concerned with the structure of the monochromatic azimuthally small-scale MHD waves in an axially symmetric magnetosphere. At present much work is being done on this subject. First of all, a number of publications (Southwood, 1977; Walker, 1987; Taylor and Walker, 1987; Ohtani et al., 1989 a,b; Miura et al., 1989) study the structure of the waves with the inclusion of such factors as a dependence of the Alfvén $A$ and sound $s$ velocities on coordinates, curvature of magnetic field lines, and plasma pressure gradient. However, all those papers assume (explicitly or implicitly) that the condition $m \gg 1$ involves the smallness of the azimuthal wavelength compared with the radial one, which permits the initial system of MHD equations to be simplified considerably by bringing it to a system of ordinary differential equations in longitudinal coordinate. The solution of this system determines the longitudinal structure of the oscillations, but with such an approach their transverse (or, more precisely, radial) structure remains unknown.

Both longitudinal and transverse structure of MHDwaves were studied in Leonovich and Mazur (1993) (hereinafter Paper I) which contains a theory discribing these waves in a cold plasma with finite curvature of field lines. A specific feature of the Alfvén waves in a curved magnetic field is the discrepancy between poloidal and toroidal frequencies of field line oscillations which is the reason for a transversal dispersion of Alfvén waves (Leonovich and Mazur, 1990). It leads to the following picture of their structure according to Paper I. There are two types of distinguished magnetic surfaces: poloidal where the wave frequency $\omega$ coincides with the frequency of poloidal eigenoscillations of the magnetosphere, and toroidal where $\omega$ coincides with the toroidal 
eigenfrequency. The transparency region of the Alfvén wave is bounded by these magnetic surfaces. The wave is produced near the poloidal surface and propagates farther away toward the toroidal surface where it is totally absorbed. Wave field singularity occurs only on the toroidal resonance surface. In the course of the propagation the polarization of the Alfvén wave changes from radial (poloidal) to azimuthal (toroidal). An extension of this theory is reported by Leonovich and Mazur (1995a,b) and Klimushkin et al. (1995). This theory is, we believe, a realistic theory for small-scale hydromagnetic waves in cold regions of the magnetosphere; however, the main merit of Paper $I$ is a mathematical theory of WKB-approximation for a two-dimensionally inhomogeneous plasma.

Our study can be considered to be a generalization of Paper I for the case of a finite-beta magnetosphere. The mathematical formalism of our paper is based on a method developed in Paper I. We found that in both a hot and cold plasma the wave propagates from the poloidal to the toroidal surface. But there are many differences in structure of the MHD-waves in a hot plasma and a cold one. The transparency region adjacent to SMS resonance [where the mode has a singularity according to Mond et al. (1990), and Cheng et al. (1993)] emerges. The Alfvén transparency region known from Paper I is found to be much wider in a hot plasma compared with a cold plasma. While in a cold plasma the poloidal surface is always nearer to the Earth than the toroidal one, in a finite-beta plasma the situation can be the opposite.

The objectives of our paper are quite similar to those of Vetoulis and Chen (1994). However, it was assumed in that paper that the plasma is an incompressible fluid, which at once removes the magnetosonic branch of the oscillations. These authors focused their attention on the study of a region where the mode on both sides is bounded by the poloidal surfaces (such a region, called the Alfvén resonator, can occur in a region of nonmonotonic variation of plasma pressure; such a possibility was also explored by Leonovich and Mazur, 1995a). Note that the situation when the transparency region is bounded on one side by the poloidal surface, and by the resonance surface on the other, is not considered by Vetoulis and Chen (1994). However, this case looks to be most general. It is precisely this case which is considered in our paper.

The paper is organized as follows. Sections 2-3 derive the governing equations in the model equilibrium. Boundary conditions on the ionosphere for functions describing the Alfvén wave and magnetosound are derived in Sect. 4. In Sect. 5 we consider the particular case of oscillations of the plasma of low but finite pressure $(0<\beta \ll 1)$ and the applicability conditions for a cold plasma approximation. In Sects. 6-9 we apply the transverse WKB-method to governing equations and the properties of the poloidal and resonance surfaces. In Sect. 10 we consider the structure of hydromagnetic waves in the vicinity of these surfaces. Main results of this study are summarized in Sect. 11, where we give a full picture of the oscillations, and compare our results with findings from other work. Finally, some mathematical manipulation omitted from Sect. 3.1, is presented in the Appendix.

\section{Model equilibrium}

The only restriction imposed on the magnetospheric plasma in our treatment is its axial symmetry, implying that all equilibrium quantities - plasma density $\rho_{0}$, pressure $P_{0}$, and magnetic field $\mathbf{B}_{0}-$ are independent of the azimuthal coordinate. The plasma equilibrium condition in a magnetic field is known to be represented as

$\nabla P_{0}=(4 \pi)^{-1} \mathbf{J}_{0} \times \mathbf{B}_{0}$.

We introduce the designation here:

$\mathbf{J}_{0} \equiv \nabla \times \mathbf{B}_{0}=\frac{4 \pi}{c} \mathbf{j}_{\perp}$,

where $\mathbf{j}_{\perp}$ is the density of transverse current flowing through the magnetosphere; for brevity's is sake, $J_{0}$ will be called simply the "current" in what follows. The equilibrium condition may also be rewritten as:

$\nabla P_{0}+\nabla_{\perp} \frac{B_{0}^{2}}{8 \pi}=\frac{B_{0}^{2}}{4 \pi R} \boldsymbol{n}$,

where $\nabla_{\perp}$ is the transverse nabla-operator, $R$ is the radius of curvature of a field line, and $\boldsymbol{n}$ is a normal to it. The invariability of pressure along field lines follows already from Eqs. (1) and (3): $\nabla P_{0}=\nabla_{\perp} P_{0}$.

We now introduce an orthogonal coordinate system "tied" to the magnetic field. In this system $x^{1}, x^{2}$, and $x^{3}$ are, respectively, the radial, azimuthal, and longitudinal (along the geomagnetic field) coordinates. The coordinate surfaces $x^{1}=$ const . coincide with magnetic shells (constant-pressure surfaces), the coordinate $x^{2}$ determines a field line on a given shell, and the coordinate $x^{3}$ defines a point on a given field line. A linear element of length is expressed as:

$d s^{2}=g_{1}\left(d x^{1}\right)^{2}+g_{2}\left(d x^{2}\right)^{2}+g_{3}\left(d x^{3}\right)^{2}$.

Here $g_{i} \equiv g_{i i}\left(x^{1}, x^{2}\right)$ are diagonal components of the metric tensor (all the others are zero because of orthogonality); $g_{i}$ are independent of $x^{2}$ because of axial symmetry. The metric tensor determinant is $g=g_{1} g_{2} g_{3}$. In our theory an important role is played by the quantity defined as:

$p=\left(g_{2} / g_{1}\right)^{1 / 2}$.

Since the field lines are directed along the coordinate lines $x^{3}$, only the longitudinal component of the geomagnetic field is nonzero, which equals $B_{0}^{3}$ in the counter-variance basis and $B_{03}$ in the co-variance basis; and $B_{0}=\sqrt{g_{3}} B_{0}^{3}$ is its "physical" value. In view of the axial symmetry, from Eq. (2) we find that the current flows along the azimuth, and, as follows from Eq. (1), it can flow in the east- or westward direction depending on the direction of $\nabla P_{0}-$ Earthward or anti-Earthward. Equilibrium conditions now become: 


$$
\begin{aligned}
& \partial_{1} P_{0}=\frac{\sqrt{g_{1}}}{4 \pi} J_{0} B_{0}, \\
& J_{0}=-\sqrt{\frac{g_{2}}{g}} \partial_{1} \sqrt{g_{3}} B_{0}, \\
& \partial_{1} P_{0}+\partial_{1} \frac{B_{0}^{2}}{8 \pi}=-\frac{B_{0}^{2}}{4 \pi R} \sqrt{g_{1}} .
\end{aligned}
$$

Knowing that $\partial_{3} P_{0}=0$ and $\operatorname{div} \mathbf{B}=0$, using Eqs. (4) and (5) it is easy to show the invariability of the countervariance component of current along a field line

$\partial_{3} J_{0}^{2}=\partial_{3} \frac{J_{0}}{\sqrt{g_{2}}}=0$.

In addition, considering that the radial component is directed anti-Earthward, toward an attenuation of the magnetic field, from Eqs. (4)-(6) we obtain a useful inequality:

$J_{0}+\frac{B_{0}}{R}=-\frac{1}{\sqrt{g_{1}}} \partial_{1} B_{0}>0$.

\section{Derivation of the system of wave equations}

We now consider the hydromagnetic wave in a hot inhomogeneous plasma with a magnetic field. Next it will be assumed that all perturbed quantities have the form

$\boldsymbol{\xi}\left(x^{1}, x^{2}, x^{3}, t\right)=\boldsymbol{\xi}\left(x^{1}, x^{3}\right) \exp \left(-i \omega t+i k_{2} x^{2}\right)$.

From the basis equations of ideal one-fluid MHD it is possible to obtain a linearized equation for plasma displacement $\xi$ in the wave (see, for example, Kadomtsev, 1963):

$$
\begin{aligned}
-\rho_{0} \omega^{2} \boldsymbol{\xi}= & \nabla\left(\boldsymbol{\xi} \cdot \nabla P_{0}+\gamma P_{0} \operatorname{div} \boldsymbol{\xi}\right) \\
& +\frac{1}{4 \pi} \mathbf{J}_{0} \times \operatorname{curl}\left[\boldsymbol{\xi} \times \mathbf{B}_{0}\right] \\
& -\frac{1}{4 \pi} \mathbf{B}_{0} \times \operatorname{curl} \operatorname{curl}\left[\boldsymbol{\xi} \times \mathbf{B}_{0}\right]
\end{aligned}
$$

(here $\gamma$ is the ratio of specific heats). With a knowledge of the displacement vector, it is possible express the wave's electric field $\mathbf{E}=\left(E_{1}, E_{2}, 0\right)$ in terms of the frozen-in condition:

$\boldsymbol{\xi}_{\perp}=-\frac{i c}{\omega} B_{0}^{-2} \mathbf{B}_{0} \times \mathbf{E}$

(the longitudinal component of the wave's electric field is zero because of ideal plasma conductivity). Given E, the Maxwell equation

$\mathbf{B}=-\frac{i c}{\omega} \nabla \times \mathbf{E}$

is used to represent three components of the wave's magnetic field.

\subsection{Identifying the Alfvén and magnetosound branches of oscillations}

Thus, using three components of the displacement vector $\left(\xi_{1}, \xi_{2}, \xi_{3}\right)$ it is possible to derive all information about the wave's behavior. However, a different choice of the variables (Klimushkin, 1994) is, we believe, more convenient: three functions, one of which describes, when passing to the limit of a homogeneous plasma, the Alfvén wave, with the other two describing two branches of magnetosound. Here we use a generally accepted definition of the Alfvén wave in a cold plasma as the wave, whose two-dimensional electric field is a potential one, i.e., it is expressed as:

$\mathbf{E}_{A}=-\nabla_{\perp} \Phi$

For both magnetosound branches, the two-dimensional electric field is a vortex one:

$\mathbf{E}_{S}=\nabla_{\perp} \times \Psi, \quad \Psi=\left(0,0, \Psi_{3}\right)$.

In the general case of an inhomogeneous plasma we may put

$\mathbf{E}=-\nabla_{\perp} \Phi+\nabla_{\perp} \times \Psi, \quad \Psi=\left(0,0, \Psi_{3}\right)$,

where, by analogy with a homogeneous plasma, $\Phi$ and $\Psi$ will be taken to be variable, which describe, respectively, the Alfvén wave and magnetosound. In a component-wise representation Eq. (13) has the form:

$\mathbf{E}_{1,2}=-\partial_{1,2} \Phi \pm \frac{g_{1,2}}{\sqrt{g}} \partial_{2,1} \Psi$

(from this point on $\Psi \equiv \Psi_{3}$ ). From Eqs. (12), (13), and (14) we obtain the components of the wave's magnetic field:

$B_{1,2}=\mp \frac{i c}{\omega} \frac{g_{1,2}}{\sqrt{g}}\left(\partial_{2,1} \partial_{3} \Phi \pm \partial_{3} \frac{g_{2,1}}{\sqrt{g}} \partial_{1,2} \Psi\right)$

$B_{3}=\frac{i c}{\omega} \frac{g_{3}}{\sqrt{g}} \Delta_{\perp} \Psi$

where the operator

$\Delta_{\perp}=\partial_{1} \frac{g_{2}}{\sqrt{g}} \partial_{1}+\partial_{2} \frac{g_{1}}{\sqrt{g}} \partial_{2}$

has the meaning of a transverse Laplacian. The quantities $\Phi$ and $\Psi$ will be referred to as potential in what follows. We will be using the quantity $\chi$ proportional to a longitudinal displacement of the plasma in the wave, as the third variable:

$\chi=\frac{i \omega}{c} \xi_{3}$

We also introduce a special designation for wave displacement divergence:

$i k_{2} \theta=\frac{i \omega}{c} \operatorname{div} \boldsymbol{\xi}$.

By expressing the displacement in terms of the potentials in accordance with Eqs. (11), (14), (16), and (17), we get 


$$
\begin{aligned}
\theta=\frac{1}{i \sqrt{g} k_{2}}\left(\partial_{3} \frac{\sqrt{g}}{g_{3}} \chi\right. & +\alpha i k_{2} \Phi \\
& \left.+\frac{g_{2}}{\sqrt{g}} \alpha \partial_{1} \Psi+\frac{\sqrt{g_{3}}}{B_{0}} \Delta_{\perp} \Psi\right),
\end{aligned}
$$

where it is designated

$\alpha=\partial_{1} \frac{\sqrt{g_{3}}}{B_{0}}=\frac{\sqrt{g_{1} g_{3}}}{B_{0}}\left(\frac{J_{0}}{B_{0}}+\frac{2}{R}\right)>0$

[the relationships of Eqs. (5), (6), and (9) are used here]. In lieu of the vector equation (10), we get the system

$$
\begin{aligned}
& \frac{\omega^{2}}{s^{2}} \chi=-i k_{2} \partial_{3} \theta \\
& -\partial_{1} \hat{L}_{T}(\omega) \partial_{1} \Phi+k_{2}^{2} \hat{L}_{P}(\omega) \Phi+i k_{2}\left(\partial_{1} \frac{\omega^{2}}{A^{2}}\right) \Psi \\
& -2 i k_{2} \partial_{3} \frac{\partial_{3} \ln p}{g_{3}} \partial_{1} \Psi=-\frac{\sqrt{g_{3}} J_{0}}{\sqrt{g_{2}} B_{0}} i k_{2} \Delta_{\perp} \Psi \\
& +\frac{4 \pi}{\sqrt{g}} k_{2}^{2} \gamma P_{0} \alpha \theta-\alpha i k_{2} \frac{J_{0}}{\sqrt{g_{2}}}\left(i k_{2} \Phi+\frac{g_{2}}{\sqrt{g}} \partial_{1} \Psi\right), \\
& {\left[\partial_{1} \frac{g_{2}}{\sqrt{g}} \hat{L}_{P}(\omega) \frac{g_{2}}{\sqrt{g}} \partial_{1}-k_{2}^{2} \frac{g_{1}}{\sqrt{g}} \hat{L}_{T}(\omega) \frac{g_{1}}{\sqrt{g}}\right] \Psi} \\
& +\Delta_{\perp} \frac{g_{3}}{\sqrt{g}} \Delta_{\perp} \Psi+i k_{2}\left(\partial_{1} \frac{\omega^{2}}{A^{2}}\right) \Phi \\
& +\left(\partial_{1} \frac{g_{2}}{\sqrt{g}} \partial_{3} \frac{g_{1}}{\sqrt{g}} i k_{2}-i k_{2} \frac{g_{1}}{\sqrt{g}} \partial_{3} \frac{g_{2}}{\sqrt{g}} \partial_{1}\right) \partial_{3} \Phi \\
& =\left(-\partial_{1} \frac{4 \pi p}{B_{0}} \partial_{1}+k_{2}^{2} \frac{4 \pi}{p B_{0}}\right) \gamma P_{0} \theta+\partial_{1} \frac{J_{0}}{\sqrt{g_{1} B_{0}}} \Delta_{\perp} \Psi \\
& +\left(-\partial_{1} \frac{g_{2}}{\sqrt{g}} \sqrt{\frac{g_{2}}{g_{3}}} \frac{J_{0}}{B_{0}} \partial_{1}+k_{2}^{2} \frac{g_{1}}{\sqrt{g}} \sqrt{\frac{g_{2}}{g_{3}}} \frac{J_{0}}{B_{0}}\right) \\
& \times\left(i k_{2} \Phi+\frac{g_{2}}{\sqrt{g}} \partial_{1} \Psi\right) .
\end{aligned}
$$

(some details of the mathematics are given in the Appendix). Here $s=\sqrt{\gamma P_{0} / \rho_{0}}$ is the sound velocity, and $A=B_{0} / \sqrt{4 \pi \rho_{0}}$ is the Alfvén velocity; the operators

$\hat{L}_{T}(\omega)=\partial_{3} \frac{g_{2}}{\sqrt{g}} \partial_{3}+\frac{\sqrt{g}}{g_{1}} \frac{\omega^{2}}{A^{2}}$,

$\hat{L}_{P}(\omega)=\partial_{3} \frac{g_{1}}{\sqrt{g}} \partial_{3}+\frac{\sqrt{g}}{g_{2}} \frac{\omega^{2}}{A^{2}}$

will be referred to, respectively, as toroidal and poloidal. The system of Eqs. (21)-(24), supplemented with appropriate boundary conditions, completely describes hydromagnetic oscillations of a hot plasma in an axisymmetric magnetic field.

To analyze the entire system, we wish above all else to note that it obviously follows from Eq. (21) that in a cold plasma $(s=0)$ at a fixed frequency $\omega \neq 0$ no longitudinal displacement is present: $\chi=0$. Further, in a homogeneous medium it is possible to introduce a Cartesian system, in which all components of the metric tensor are 1 , and put $\partial_{1}=i k_{1}, \partial_{3}=i k_{3}$. Then Eq. (22) reduces to a dispersion equation of the Alfvén wave: $\omega^{2}=k_{3}^{2} A^{2}$

and the system of Eqs. (21), (23) reduces to an algebraic equation for $\omega^{2}$

$\omega^{4}-\omega^{2} k^{2}\left(s^{2}+A^{2}\right)+s^{2} A^{2} k_{z}^{2} k^{2}=0$

(where $k^{2}=k_{x}^{2}+k_{y}^{2}+k_{z}^{2}$ ), which has two roots, one of which is the dispersion equation for FMS, and the other for SMS. In the case of $k_{\perp} \gg k_{\|}$the second is written as:

$\omega^{2}=k_{\|}^{2} \frac{s^{2} A^{2}}{s^{2}+A^{2}}\left[1+O\left(\frac{k_{\|}^{2}}{k_{\perp}^{2}}\right)\right]$.

\subsection{Reduction of the system of equations for the case $m \gg 1$}

The system Eqs. (21)-(23) is, of course, too complicated for it to be used directly. Yet it can be simplified if only oscillations with $m \gg 1$ are considered. If the azimuthal angle $\phi$ is taken to be the coordinate $x^{2}$, then $k_{2}=m$. We introduce here the quantity $L$, a typical scale of variation of equilibrium magnetospheric parameters (a value about the size of the magnetosphere). From $m \gg 1$ we then get the condition for a characteristic azimuthal wavelength

$\lambda_{a} \sim \frac{L}{m} \ll L$

As far as the longitudinal wavelength is concerned, its value for the fundamental harmonics of the standing wave $(N \sim 1)$ is of the same order of magnitude as $L$, i.e., we obtain:

$\frac{1}{\sqrt{g_{3}}}\left|\partial_{3}\right| \ll \frac{1}{\sqrt{g_{2}}} k_{2}$

(here the differential operator is thought of as exerting an influence upon disturbed quantities only).

Next, we compare the azimuthal wavelength with a characteristic wavelength in radial coordinate $\lambda_{r}$. In Walker (1987) it is assumed that $\lambda_{r} \gg \lambda_{a}$. A high value of $m$ is a formal basis for this assumption. However, this assumption does not take into account the possibility that a characteristic value of the radial component of wave vector $k_{1}$ increases with increasing $m$, i.e., the characteristic wavelength $\lambda_{r}$ can decrease. It is shown in Paper I that the inequality $k_{2} \gg \partial_{1}$ holds only near the poloidal surface, whereas with distance from it $\lambda_{r}$ became to be the same order as $\lambda_{a}$ and near the resonance surface the wave has a singularity $\left(\lambda_{r} \ll \lambda_{a}, k_{1} \rightarrow \infty\right)$. There is good reason to believe that in the case of finite-beta plasma we have an analogous situation.

Based on the preceding we suppose that in general terms

$\lambda_{r} \sim \lambda_{a} \sim \frac{L}{m} \sim \lambda_{\perp} \ll L$

that is we should keep derivatives $\partial_{1}$ and the multiplier $k_{2}$ in the equations, i.e.,

$L \hat{\partial}_{1} \sim m, \quad \hat{k}_{2} \sim \hat{\partial}_{1}$, 
where $\hat{k}_{2}=\sqrt{g_{2}} m$. It is apparent that the assumptions Eqs. (27), (28) do not rule out the possibility that near some surfaces (poloidal and resonance) $k_{2} \gg \partial_{1}$ or $k_{2} \ll \partial_{1}$ (in the vicinity of these surfaces it is possible to make further simplifications of the equations; see Sect. 10). At the same time the inequalities of Eqs. (27), (28) permit us to ignore the transverse derivatives of equilibrium plasma parameters as compared to the derivatives of disturbed fields.

Let us consider terms with maximum degrees of $m$ involved in Eqs. (21)-(23). In view of Eq. (28), this system yields order-of-magnitude relationships between the potentials:

$\chi \sim m \Phi \sim m^{2} \Psi$.

We now rewrite this system by leaving only terms with maximum degrees of $m$ in each equation

$$
\begin{gathered}
\frac{\omega^{2}}{s^{2}} \chi+\partial_{3} \frac{1}{\sqrt{g_{3}}} \partial_{3} \frac{\sqrt{g}}{g_{3}} \chi=-\partial_{3} \frac{\Delta_{\perp} \Psi}{\sqrt{g_{\perp}} B_{0}}-\partial_{3} \frac{\alpha i k_{2}}{\sqrt{g}} \Phi, \\
-\partial_{1} \hat{L}_{T}(\omega) \partial_{1} \Phi+k_{2}^{2} \hat{L}_{P}(\omega) \Phi=-\frac{\sqrt{g_{3}} J_{0}}{\sqrt{g_{2}} B_{0}} i k_{2} \Delta_{\perp} \Psi \\
+k_{2}^{2} \alpha\left(\frac{J_{0}}{\sqrt{g_{2}}}+\frac{4 \pi \gamma P_{0} \alpha}{\sqrt{g}}\right) \Phi-\frac{4 \pi i k_{2} \gamma P_{0} \alpha}{B_{0} \sqrt{g_{\perp}}} \Delta_{\perp} \Psi \\
\quad-\frac{4 \pi i k_{2} \gamma P_{0} \alpha}{\sqrt{g}} \partial_{3} \frac{\sqrt{g}}{g_{3}} \chi, \\
\Delta_{\perp} \frac{g_{3}}{\sqrt{g}} \Delta_{\perp} \Psi=-\Delta_{\perp} \frac{4 \pi \sqrt{g_{3}}}{B_{0}} \frac{\gamma P_{0}}{\sqrt{g}}\left(\partial_{3} \frac{\sqrt{g}}{g_{3}} \chi+\alpha i k_{2}\right. \\
\left.+\frac{\sqrt{g_{3}}}{B_{0}} \Delta_{\perp} \Psi\right)-\Delta_{\perp} \frac{\sqrt{g_{3}} J_{0}}{\sqrt{g_{2}} B_{0}} i k_{2} \Phi .
\end{gathered}
$$

Equation (32) may be treated as an ordinary differential equation of the form

$$
\frac{g_{2}}{\sqrt{g}} \partial_{1} \partial_{1} u-\frac{g_{1}}{\sqrt{g}} k_{2}^{2} u=0
$$

for the function

$$
\begin{aligned}
u= & \frac{g_{3}}{\sqrt{g}} \frac{s^{2}+A^{2}}{A^{2}} \Delta_{\perp} \Psi \\
& +\frac{s^{2}}{A^{2}} \frac{B_{0}}{\sqrt{g_{\perp}}}\left(\partial_{3} \frac{\sqrt{g}}{g_{3}} \chi+\alpha i k_{2} \Phi\right)+\sqrt{g_{1}} \frac{J_{0}}{B_{0}} i k_{2} \Phi,
\end{aligned}
$$

of which a typical scale of the solution in $x^{1}$ has the order of magnitude $\lambda_{r} \sim L / m$ [in agreement with Eq. (27)]. This equation has two untrivial solutions: one of them increases when $x^{1} / \lambda_{r} \rightarrow \infty$, and the other increases when $x^{1} / \lambda_{r} \rightarrow-\infty$. However, a natural boundary condition is the absence of any increase of the potentials when $x^{1} \rightarrow \infty$

$|\Phi, \Psi, \chi|_{x^{1} \rightarrow \pm \infty}<\infty$.

The only solution (compatible with this condition) is the trivial solution $u=0$, i.e.,

$$
\begin{aligned}
\Delta_{\perp} \Psi= & -\frac{v_{S}^{2}}{A^{2}} \frac{B_{0}}{\sqrt{g_{3}}}\left(\partial_{3} \frac{\sqrt{g}}{g_{3}} \chi+\alpha i k_{2} \Phi\right) \\
& -\frac{v_{S}^{2}}{s^{2}} \sqrt{g_{1}} \frac{J_{0}}{B_{0}} i k_{2} \Phi
\end{aligned}
$$

where

$v_{S}^{2}=\frac{s^{2} A^{2}}{s^{2}+A^{2}}$

is the slow magnetosound velocity squared. On substituting Eq. (34) into Eq. (19), we obtain:

$i k_{2} \sqrt{g} \theta=\frac{v_{S}^{2}}{s^{2}} \partial_{3} \frac{\sqrt{g}}{g_{3}} \chi+\alpha_{1} \frac{v_{s}^{2}}{s^{2}} i k_{2} \Phi$,

where $\alpha_{1}=\frac{2 \sqrt{g \cdot g_{3}}}{B_{0} R}$. Using Eqs. (34), (35) we rewrite Eqs. (30), (31) in Hermitian form:

$\hat{L}_{S}(\omega) \Theta=\alpha_{0} \omega \Phi$,

where

$\hat{L}_{S}(\omega)=\sqrt{g} s^{2} \frac{\omega^{2}}{v_{S}^{2}}+\partial_{3} s^{2} \frac{\sqrt{g}}{g_{3}} \partial_{3}$

is the SMS mode operator, and

$\Theta=\frac{\sqrt{4 \pi \gamma P_{0}}}{\omega} \theta, \quad \alpha_{0}=\sqrt{4 \pi \gamma P_{0}} \alpha_{1}$

[when obtaining Eq. (36), we have additionally multiplied Eq. (30) by $\left(s^{2} / g_{3}\right)$ and then let it be acted upon by the operator $\left.\partial_{3}\right]$ and

$$
-\partial_{1} \hat{L}_{T}(\omega) \partial_{1} \Phi+k_{2}^{2} \hat{L}_{P}(\omega) \Phi-k_{2}^{2} \frac{\alpha_{1} J_{0}}{\sqrt{g_{2}}} \Phi=k_{2}^{2} \alpha_{0} \omega \Theta
$$

[Eq. (20) is used here]. Thus, we have passed from the variables $\Phi, \Psi, \chi$ to the variables $\Phi, \Psi, \Theta$; the latter set of variables is more convenient because algebraic, rather than differential operators turn out to appear in righthand sides of Eqs. (36), (37). These equations are the governing equations used for all the wave analysis presented in the paper.

\subsection{Discussion of the system of wave equations}

Consider some properties of the system of Eqs. (36), (37). First and foremost we turn to the case of a homogeneous plasma, for which it is possible to choose a coordinate system $\left(g_{i}=1, i=1,2,3\right)$ and put $\partial_{1}=i k_{1}, \partial_{3}=i k_{3}$. Then Eq. (36) becomes

$s^{2}\left(\frac{\omega^{2}}{v_{S}^{2}}-k_{3}^{2}\right) \Theta=0$.

This equality is satisfied when $\Theta \neq 0$ if the dispersion equation of the SMS wave $\omega^{2}=k_{3}^{2} v_{S}^{2}$ is valid. Similarly, Eq. (37) is brought to the form

$\left(k_{1}^{2}+k_{2}^{2}\right)\left(\frac{\omega^{2}}{A^{2}}-k_{3}^{2}\right) \Phi=0$, 
implying that the dispersion equation of the Alfvén wave $\omega^{2}=k_{3}^{2} A^{2}$ is satisfied. Therefore, we will call Eqs. (36) and (37), respectively, the magnetosound and Alfvén equations. Equation (34) in the homogeneous case turns out to be the condition for coupling between a longitudinal and transverse displacement in the MHD wave. We did not make any assumptions about FMS, but we found that the condition $m \gg 1$ automatically entails its absence. For fast magnetosound with $m \gg 1$ the magnetosphere is opaque; see also Walker (1987), Leonovich and Mazur (1990).

In the inhomogeneous case, in the presence of a curvature of field lines, the Alfvén and magnetosound equations turned out to be coupled, that is consistent with the results of Southwood and Saunders (1985), Walker (1987), and Ohtani et al. (1989a).

In the particular case of a cold plasma Eq. (37) becomes

$\partial_{1} \hat{L}_{T}(\omega) \partial_{1} \Phi-k_{2}^{2} \hat{L}_{P}(\omega) \Phi=0$.

The structure of the Alfvén wave described by this equation was studied in detail in Paper I. Note that Eq. (36) when $\beta=0$ reduces to the equality $\sqrt{g} \Theta=\alpha_{0} \Phi$, implying, because of Eq. (35), that $\chi=0$.

Using the system of Eqs. (34), (36), (37) it is also possible to study the behavior of plasma pressure $\delta P$ of the hydromagnetic wave that in a general case is expressed by the formula

$$
\delta P=-\xi \cdot \nabla P_{0}-\gamma P_{0} \operatorname{div} \boldsymbol{\xi}
$$

(see, for example, Kadomtsev, 1963). By expressing $\xi$ in terms of the potentials, $\nabla P_{0}$ from Eq. (5), div $\xi$ from Eqs. (18) and (35) and $B_{3}$ from Eqs. (15) and (35), we obtain:

$\delta P=-\frac{c k_{2}}{\omega}\left(\gamma P_{0} \Theta+\frac{J_{0}}{\sqrt{g_{2}}} i k_{2} \Phi\right)=-\frac{B_{0} B_{3}}{4 \pi \sqrt{g_{3}}}$.

\section{Boundary conditions on the ionosphere}

In a most general form, boundary conditions on the ionosphere are represented as (Paper I):

$$
\left(E_{j} \pm \frac{i c^{2} \cos \alpha}{4 \pi \omega \Sigma_{p}} \partial_{3} E_{j}\right)_{ \pm}=\frac{\cos \alpha_{ \pm}}{\sum_{p}^{ \pm}} \partial_{j} I_{\|}^{ \pm}
$$

$(j=1,2)$. Here the + and - signs correspond to points at which a field line intersects the ionosphere $\left(x^{3}=x_{ \pm}^{3}\right), \alpha$ is the angle between a field line and a vertical to the ionosphere, $\Sigma_{p}^{ \pm}$is integral Pedersen conductivity of the ionosphere, and $I_{\|}^{ \pm}$is a field-aligned current function satisfying the equation

$\Delta_{\perp}^{ \pm} I_{\|}^{ \pm}=j_{\|}^{ \pm}$,

where $\Delta_{\perp}^{ \pm}$is the transverse Laplacian Eq. (16) when $x^{3}=x_{ \pm}^{3}$, and $j_{\|}$is the density of external field-aligned current in the ionosphere. According to Paper I, $j_{\|}$can be excited by gravity waves in the ionosphere and can be the source for transversally small-scale hydromagnetic waves; the second term on the left-hand side of Eq. (40) describes the dissipation of the mode on the ionosphere. We now introduce the designations:

$a=\frac{c^{2} \cos \alpha}{4 \pi \omega \Sigma_{p}}, \partial_{j} b=\frac{\cos \alpha}{\Sigma_{p}} \partial_{j} I_{\|}$

The effects of dissipation $a$ and currents $b$ are important only when the structure near the resonance and poloidal surfaces is studied (Sect. 10) but in WKB-approximation (Sects. 6-9) we can use perfectly reflecting ionospheric boundary conditions. Now we rewrite Eq. (40) in terms of the potentials $\Phi, \Psi$. In view of the transverse smallscale character of the oscillations, we obtain:

$i k_{2}\left(\frac{g_{1}}{\sqrt{g}} \Psi \pm i a \partial_{3} \frac{g_{1}}{\sqrt{g}} \Psi\right)_{ \pm}=\partial_{1}\left(\Phi \pm i a \partial_{3} \Phi+b\right)_{ \pm}$,

$\partial_{1}\left(\frac{g_{2}}{\sqrt{g}} \Psi \pm i a \partial_{3} \frac{g_{2}}{\sqrt{g}} \Psi\right)_{ \pm}=-i k_{2}\left(\Phi \pm i a \partial_{3} \Phi+b\right)_{ \pm}$

For transversally small-scale oscillations, the estimation of Eq. (29) holds true. This means that in the main order in Eq. (41 a, b) terms containing $\Psi$ may be neglected. We will also make a further simplifying assumption: we restrict ourselves to waves for which the contribution of the dissipation $a$ and external currents $b$ is unimportant. Terms containing these quantities will be referred to the same order of smallness as $\Psi$, i.e., we put

$\Phi=\Phi^{(0)}+\Phi^{(1)}, \quad\left|\Phi^{(1)}\right| \ll\left|\Phi^{(0)}\right|$

By linearizing Eq. (41 a,b), we obtain in the main order the boundary condition for $\Phi^{(0)}$ :

$\Phi_{ \pm}^{(0)}=0$

In the next order of perturbation theory we obtain the boundary condition for the correction $\Phi^{(1)}$ :

$\Phi_{ \pm}^{(1)}=\mp\left(i a \partial_{3} \Phi^{(0)}\right)_{ \pm}+b_{ \pm}$

[when obtaining this expression, we let $a$ and $b$ of Eq. (41) be acted upon, respectively, by the operators $\left(g_{2} / \sqrt{g}\right) \partial_{1}$ and $\left(g_{1} / \sqrt{g}\right) i k_{2}$, subtracted the resulting expressions from each other and "cancelled out" the Laplacian in much the same manner as when deriving Eq. (34)]. As is evident, the boundary conditions for $\Phi$ turn out to contain no function $\Psi$.

It is also easy to obtain boundary conditions for the functions $\Psi$ and $\Theta$. To accomplish this, they may also be represented as

$\Psi=\Psi^{(0)}+\Psi^{(1)}, \quad\left|\Psi^{(1)}\right| \ll\left|\Psi^{(0)}\right|$,

$\Theta=\Theta^{(0)}+\Theta^{(1)}, \quad\left|\Theta^{(1)}\right| \ll\left|\Theta^{(0)}\right|$

(the corrections $\Psi^{(1)}$ and $\Theta^{(1)}$ take into account the contribution of the dissipation and external currents). We obtain boundary conditions for $\Psi$ directly from Eq. (41 a,b):

$\Psi_{ \pm}^{(0)}=0$ 


$$
\Psi_{ \pm}^{(1)}=\mp\left(i a \partial_{3} \Psi^{(0)}\right)_{ \pm}
$$

To obtain boundary conditions for $\Theta$, we must invoke Eq. (35) Eqs. (44), (45) just obtained. We get

$\Theta_{ \pm}^{(0)}=0$

$\Theta_{ \pm}^{(1)}=\mp\left(i a \partial_{3} \Theta^{(0)}\right)_{ \pm}$.

Note that in the boundary conditions the functions $\Phi$, $\Psi$, and $\Theta$ have split.

Thus, we have obtained the boundary conditions on the ionosphere for the variables $\Phi, \Psi, \Theta$ [Eqs. (42)-(47)]. Together with the boundary conditions in radial coordinate Eq. (33) and Eqs. (35)-(37), they constitute the problem for hydromagnetic waves in axisymmetric plasma of arbitrary pressure.

\section{Alfvén oscillations of low pressure plasma, and the validity range of the cold plasma approximation}

It is commonly believed that the outer magnetospheric regions (say, the MacIlwain parameter $L>5$ ) are "hot", while the inner regions are "cold" (see, e.g., Hargeaves, 1979). A review of observational data on pulsations with $m \gg 1$ in the inner regions is contained in the paper of Takahashi and Anderson (1992). In this section we take up the question of the character of plasma oscillations with finite but small values of $\beta \ll 1$ with an eye also to revealing the validity range of the cold plasma approximation for investigating the Alfvén waves used in some earlier work, including Paper I.

In the case $\beta \ll 1$ the Alfvén and magnetosonic resonances are spaced greatly. In a region reasonably far away from the SMS-resonance, the quantity $\Theta$ is representable as

$\Theta=\Theta_{0}+\Theta_{1}$,

where $\Theta_{0}$ is the solution corresponding to $\beta=0$, and $\Theta_{1}$ is a correction caused by a small difference of $\beta$ from unity. By linearizing the magnetosonic equation (36), in the main order we obtain:

$\Theta_{0}=\frac{\alpha_{0}}{\omega \sqrt{g}} \frac{v_{S}^{2}}{s^{2}} \Phi$

By expressing $\Theta$ from Eq. (48) and substituting it into Eq. (37), we obtain the equation

$$
-\partial_{1} \hat{L}_{T}(\omega) \partial_{1} \Phi+k_{2}^{2} \hat{L}_{P}(\omega) \Phi-k_{2}^{2} \frac{B_{0}}{\sqrt{g_{2}}} \alpha_{1} \eta \Phi=0
$$

where

$$
\eta=\frac{J_{0}}{B_{0}}+\frac{2}{R} \frac{s^{2}}{A^{2}} \text {. }
$$

Equation (49) is identical in its form to that of Alfvén oscillations of cold plasma, Eq. (38) with the substitution of the poloidal operator $\hat{L}_{P}(\omega)$ for its analogue in a warm plasma $\hat{L}_{P W}(\omega)$ :
$\hat{L}_{P W}(\omega)=\hat{L}_{P}(\omega)+\delta_{N}$

$\delta_{N}=-\frac{\alpha_{1}}{\sqrt{g_{2}}}\left(J_{0}+\frac{2 B_{0}}{R} \frac{s^{2}}{A^{2}}\right)$.

Therefore, all conclusions of the theory of Alfvén waves in cold plasma (Paper I) must also be applicable to warm plasma; however, there must arise some quantitative differences.

First of all, to the operators $\hat{L}_{P}(\omega)$ and $\hat{L}_{P W}(\omega)$ there correspond different eigenfrequencies. Let their difference be designated as $\Delta \Omega_{P N}$. It is easy to calculate this quantity using perturbation theory by considering the quantity $\delta_{N}$ in Eq. (50), proportional to $\beta$, to be a small correction. We omit intermediate developments and give the final result:

$\Delta \Omega_{P N}=\frac{1}{2 \Omega_{P N}} \oint \frac{\alpha_{1} \eta B_{0}}{\sqrt{g_{2}}} P_{N}^{2} d x^{3}$,

where $P_{N}$ is the eigenfunction of the operator $\hat{L}_{P}(\omega)$ normalized by the condition

$\oint \frac{\sqrt{g}}{g_{2}} \frac{P_{N}^{2}}{A^{2}} d x^{3}=1$.

As is apparent, on the order of magnitude

$\frac{\Delta \Omega_{P N}}{\omega} \sim \beta$.

When $\eta<0$, the sign of $\Delta \Omega_{P N}$ is negative, i.e. the poloidal surface lies nearer to the Earth compared to the cold plasma case. When $\eta>0$, the situation is the reverse: the poloidal surface approaches the toroidal surface. If $\Delta \Omega_{P N}$ exceeds the difference between the poloidal and toroidal frequencies in cold plasma, then the poloidal surface finds itself farther from the Earth compared with the toroidal surface.

Using Eq. (52) one can determine from which values of $\beta$ the magnetosphere can no longer be considered cold. Obviously, the finiteness of $\beta$ must be taken into account if

$\beta>\frac{\Delta_{N}}{L}$,

where $\Delta_{N}$ is the distance between the poloidal and toroidal surfaces in cold plasma. If $N=1$ then $\Delta_{N} / L \sim 10^{-2}-10^{-1}$ and if $N \geq 2$ then $\Delta_{N} / L \sim 10^{-3}$ (Paper I). Thus, when $\beta \sim 10^{-1}$ the finiteness of plasma pressure must be taken into account at any values of the longitudinal harmonic number. If, however, $N$ is larger than unity (beginning with $N=2$ ), then finite pressure must be taken into account even if $\beta \sim 10^{-3}$. At the same time, observed poloidally polarized oscillations have $N=2$ and $m \sim 50$ (Anderson, 1994).

\section{The transverse WKB-approximation}

When we study the MHD-waves structure in the interval between the resonance and poloidal surfaces, we use the WKB-approximation over transverse coordinates which 
was accomodated to two-dimensionally inhomogeneous plasma in Paper I.

Write the functions $\Phi$ and $\Theta$ as

$\Phi=\exp \left(i Q_{A}\right), \quad \Theta=\exp \left(i Q_{S}\right)$.

In Sect. 3 it was shown that the small-scale character of the mode in azimuth (i.e., the condition $m \gg 1$ ) implies a radial small-scale character of the mode Eqs. (27), (28) signifying that the following relationships

$\left|\frac{1}{\sqrt{g_{1}}} \partial_{1} \Phi\right| \gg\left|\frac{1}{\sqrt{g_{3}}} \partial_{3} \Phi\right|,\left|\frac{1}{\sqrt{g_{1}}} \partial_{1} \Theta\right| \gg\left|\frac{1}{\sqrt{g_{3}}} \partial_{3} \Theta\right|$

are valid. Similar relationships for the phase $Q$ follow herefrom:

$\left|\frac{1}{\sqrt{g_{1}}} \partial_{1} Q_{A, S}\right| \gg\left|\frac{1}{\sqrt{g_{3}}} \partial_{3} Q_{A, S}\right|$,

i.e., the dependence of the phase of the Alfvén and magnetosonic waves on the radial coordinate $x^{1}$ is much stronger than the dependence on the longitudinal coordinate $x^{3}$. This means that from the phases it is possible to single out main terms dependent only on $x^{1}$, i.e., in an asymptotic expansion

$Q_{A, S}=Q_{A, S}^{(0)}+Q_{A, S}^{(1)}+Q_{A, S}^{(2)}+\cdots$

the main terms $\left(Q_{A}^{(0)}\right.$ and $\left.Q_{S}^{(0)}\right)$ are independent of $x^{3}$. Thus, in the WKB-approximation the relationships are representable as

$\Phi=F \exp \left[i Q_{A}^{(0)}\right], \Theta=H \exp \left[i Q_{S}^{(0)}\right]$,

where it is designated

$F=F\left(x^{1}, x^{3}\right)=\exp \left[i Q_{A}^{(1)}\right]$,

$H=H\left(x^{1}, x^{3}\right)=\exp \left[i Q_{S}^{(1)}\right]$.

In the WKB-approximation $Q_{A, S}^{(0)} \gg 1$; therefore, the exponents in Eq. (54) describe the fast-varying functions $x^{1}$, the radially small-scale structure of the Alfvén and SMS-modes. On substituting Eq. (54) into Eq. (36), we obtain:

$\hat{L}_{S}(\omega) H=\alpha_{0} \omega F \exp \left[i Q_{A}^{(0)}-i Q_{S}^{(0)}\right]$.

The left-hand side of this equation contains only a slow dependence on $x^{1}$, while the right-hand side involves also a fast dependence entering in terms of the difference of the phases $Q_{A}^{(0)}-Q_{S}^{(0)}$. This means that the last equation can be satisfied, provided that $Q_{A}^{(0)}=Q_{S}^{(0)}$ $=Q^{(0)}$, i.e., a fast dependence on the radial coordinate in both branches is described by the same function. On this basis, we substitute Eq. (54) into Eqs. (36) and (37) and obtain, in the main order of WKB, the following system of ordinary differential equations (in coordinate $x^{3}$ )

$\hat{L}_{S}(\omega) H=\alpha_{0} \omega F$,

$k_{1}^{2} \hat{L}_{T}(\omega) F+k_{2}^{2} \hat{L}_{P}(\omega) F-k_{2}^{2} \frac{J_{0} \alpha_{1}}{\sqrt{g_{2}}} F=k_{2}^{2} \alpha_{0} \omega H$,

with the notation: $k_{1}=\partial_{1} Q_{0}$

where $k_{1}$ has the meaning of the radial component of a transverse wave vector. In view of Eq. (54), the boundary conditions of Eqs. (42), (46) may be written as

$H_{ \pm}=0$,

$F_{ \pm}=0$

(perfectly reflecting ionospheric boundary conditions).

Following Paper I, we treat the problem of Eqs. (55)(59) as a problem for eigenvalues of the quantity $k_{1}$. With a given value of $\omega$, the solutions of this problem are the functions $H=H_{N}\left(x^{1}, x^{3}, \omega\right)$ and $F=F_{N}\left(x^{1}, x^{3}, \omega\right)$, and the eigenvalue is $k_{1}=k_{1 N}\left(x^{1}, \omega\right)$. Here $N$ is the longitudinal harmonic number of the standing wave.

\section{Resonance surfaces}

A treatment of the problem given by Eqs. (55)-(59) outlined in the preceding section is natural from the physical standpoint, but a different, strictly formal treatment is possible: by considering the quantity $k_{1}$ to be given and the frequency $\omega$ to be an eigenvalue. For instance, one may put $k_{1}^{2}=\infty$. This equality is satisfied on the magnetic shells called the resonance surfaces. As pointed out already there are two such surfaces in the magnetosphere: one of them corresponds to Alfvén resonance and the other to SMS resonance (Mond et al., 1990; Cheng et al., 1993). Here we consider an eigenvalue problem for the waves in the vicinity of these surfaces.

In the limit $\hat{k}_{1} \gg \hat{k}_{2}$, from Eq. (56) we get:

$H_{N}=\frac{k_{1}^{2}}{k_{2}^{2}} \frac{1}{\alpha_{0} \omega} \hat{L}_{T}(\omega) F_{N}$.

On substituting this expression into Eq. (55), we obtain in the limit $k_{1}^{2} \rightarrow \infty$ :

$\hat{L}_{S}(\omega) \frac{1}{\alpha_{0} \omega} \hat{L}_{T}(\omega) F_{N}=0$

This equality can be satisfied in two cases only. One is when the function $F_{N}$ is an eigenfunction of the operator $\hat{L}_{T}(\omega)$ :

$\hat{L}_{T}(\omega) F_{N}=0,\left.\quad F_{N}\right|_{ \pm}=0$.

We now introduce a toroidal eigenfunction $T_{N}$ which is the solution of the problem Eq. (61) and has the normalization

$\oint \frac{\sqrt{g}}{g_{1}} \frac{T_{N}^{2}}{A^{2}} d x^{3}=1$.

Then $F_{N}=f\left(x^{1}\right) T_{N}\left(x^{1}, x^{3}\right)$, where $f\left(x^{1}\right)$ is an arbitrary function (the main order of the WKB-approximation is insufficient for defining it). Equation (61) can be satisfied provided that $\omega$ coincides with the eigen-frequency $\Omega_{T N}\left(x^{1}\right)$ of the operator $\hat{L}_{T}(\omega)$. Note that $T_{N}$ and $\Omega_{T N}$ depend on $x^{1}$ as a parameter. 
If, however, Eq. (61) does not hold, then from Eq. (60) follows the fulfillment of the condition

$\hat{L}_{S}(\omega) H_{N}=0,\left.H_{N}\right|_{ \pm}=0$.

We designate by $U_{N}$ the solution of this problem normalized as follows:

$\oint \sqrt{g} \frac{s^{2}}{A^{2}} U_{N}^{2} d x^{3}=1$.

It is obvious that $H_{N}=f\left(x^{1}\right) U_{N}\left(x^{1}, x^{3}\right)$, where $f\left(x^{1}\right)$ is an arbitrary function $x^{1}$. It follows from Eq. (63) that $\omega=\Omega_{S N}\left(x^{1}\right)$, where $\Omega_{S N}\left(x^{1}\right)$ is the eigenfrequency of the operator $\hat{L}_{S}(\omega)$.

It is easy to determine the eigenfunctions and the eigenfrequencies of the operators $\hat{L}_{T}(\omega)$ and $\hat{L}_{S}(\omega)$ by solving relevant equations in the longitudinal WKBapproximation $(N \gg 1)$, i.e., by substituting the functions $T_{N}, U_{N}$ in the form

$T_{N}, U_{N}=\exp \left[i S_{0}\left(x^{3}\right)+i S_{1}\left(x^{3}\right)\right],\left|S_{1}\right| \ll\left|S_{0}\right|$.

On substituting this expression into Eq. (61), we obtain - using boundary conditions and a normalization Eq. (62) - the following expression for $T_{N}$ :

$T_{N}=\frac{\Omega_{T N}}{\sqrt{\pi N}}\left(k_{T N} \frac{g_{2}}{\sqrt{g}}\right)^{-1 / 2} \sin \int_{x_{-}^{3}}^{x^{3}} k_{T N}\left(x^{3^{\prime}}\right) d x^{3^{\prime}}$,

where

$\Omega_{T N}=\frac{2 \pi N}{t_{A}}, t_{A}=\oint \frac{d x^{3} \sqrt{g_{3}}}{A}$

is a toroidal eigenfrequency, and

$k_{T N}=\frac{\Omega_{T N}}{A} \sqrt{g_{3}}$

is a longitudinal wave vector of the toroidal mode. In a similar spirit we obtain the expression for the magnetosonic mode:

$U_{N}=\frac{\Omega_{S N}}{\sqrt{\pi N}}\left(k_{S N} s^{2} \frac{\sqrt{g}}{g_{3}}\right)^{-1 / 2} \sin \int_{x_{-}^{3}}^{x^{3}} k_{S N}\left(x^{3^{\prime}}\right) d x^{3^{\prime}}$,

where

$\Omega_{S N}=\frac{2 \pi N}{t_{S}}, t_{S}=\oint \frac{d x^{3} \sqrt{g_{3}}}{v_{S}}$

is the magnetosonic eigenfrequency, and

$k_{S N}=\frac{\Omega_{S N}}{v_{S}} \sqrt{g_{3}}$

is a longitudinal wave vector for the SMS-mode.

Since the physical statement of the problem is such that we consider the wave with a given frequency $\omega$, we arrive at the conclusion that the equality $k_{1}^{2}=\infty$ occurs at values of $x^{1}=x_{T N}^{1}$ and $x^{1}=x_{S N}^{1}$ determined from the equations

$\omega=\Omega_{T N}\left(x^{1}\right)$ and $\omega=\Omega_{S N}\left(x^{1}\right)$.

The magnetic shells $x^{1}=x_{T N}^{1}$ and $x^{1}=x_{S N}^{1}$ will be referred to, respectively, as the toroidal and SMS- resonance surfaces. (In accordance with terminology accepted in plasma physics, resonance on the toroidal surface will also be called the Alfvén resonance).

It will be assumed in what follows that the toroidal surface lies farther from the Earth compared with the SMS-resonance surface. Indeed, the Alfvén velocity is higher than the slow magnetosound velocity $\left(A^{2}>v_{S}^{2}\right.$ $\equiv s^{2} A^{2} /\left(s^{2}+A^{2}\right)$ ). Using Eqs. (66) and (69) we conclude that $\Omega_{S N}\left(x^{1}\right)<\Omega_{T N}\left(x^{1}\right)$. Assuming that $A$ and $v_{S}$ decrease with increasing $x^{1}$ (just such is the case in most of the magnetosphere) we conclude that the magnetosonic resonance lies nearer to the Earth than the Alfvén one.

\section{Structure of the wave's transparency regions}

Our intention now is to ascertain where the wave's transparency regions (i.e., regions with $k_{1}^{2}>0$ ) are located with respect to resonance shells. To do so, we have to study the behavior of the function near the points $x_{T N}^{1}$ and $x_{S N}^{1}$, as well as determining the position of the points where the equality $k_{1}^{2}=0$ holds (poloidal surfaces).

8.1 Behavior of the function $k_{I N}^{2}\left(x^{1}\right)$ near the toroidal resonance surface

Near the toroidal surface (of Alfvén resonance), the relationship

$\left|\omega^{2}-\Omega_{T N}^{2}\right| \ll \omega^{2}$

holds. In this region a compensation of the large quantity $k_{1}^{2}$ in Eq. (56) occurs because of the smallness of the expression $\hat{L}_{T}(\omega) F_{N}$. Therefore, the solution $F_{N}$ may be represented as

$F_{N}=T_{N}+t_{N},\left|t_{N}\right| \ll\left|T_{N}\right|$,

where $T_{N}$ is the previously introduced toroidal eigenfunction, and $t_{N}$ is a small addition caused by a small deviation of $\omega$ from $\Omega_{T N}$; boundary conditions for this function have the form

$\left.t_{N}\right|_{ \pm}=0$

(perfectly reflecting ionospheric boundary conditions). Let the value of $H_{N}$ when $x^{1} \approx x_{T N}^{1}$ be represented as $H_{T N}$. On substituting Eq. (72) into Eq. (56) and linearizing, we obtain in the main order equation (61) and in the next order,

$$
\begin{aligned}
& k_{1 N}^{2} \frac{\omega^{2}-\Omega_{T N}^{2}}{A^{2}} \frac{\sqrt{g}}{g_{1}} T_{N}+k_{1 N}^{2} \hat{L}_{T}\left(\Omega_{T N}\right) t_{N} \\
& \quad+\quad k_{2}^{2} \hat{L}_{P}\left(\Omega_{T N}\right) T_{N}-k_{2}^{2} \frac{\alpha_{1} J_{0}}{\sqrt{g_{2}}} T_{N}=k_{2}^{2} \alpha_{0} \Omega_{T N} H_{T N} .
\end{aligned}
$$

We multiply this expression by $T_{N}$ and integrate along a field line "there and back". Using the Hermitian character of the operator and the normalization Eq. (62) we obtain

$k_{1 N}^{2}=-k_{2}^{2} \frac{w_{T N}}{\omega^{2}-\Omega_{T N}^{2}}$, 
where it is designated: $w_{T N}=w_{T N 1}-w_{T N 2}-w_{T N 3}$,

$$
\begin{aligned}
& w_{T N 1}=\oint T_{N} \hat{L}_{P}\left(\Omega_{T N}\right) T_{N} d x^{3}, \\
& w_{T N 2}=\oint T_{N}^{2} \frac{\alpha_{1} J_{0}}{\sqrt{g_{2}}} d x^{3}, \\
& w_{T N 3}=\Omega_{T N} \oint \alpha_{0} H_{T N} T_{N} d x^{3} .
\end{aligned}
$$

This last expression is conveniently rewritten in terms of Green's function $G_{S}\left(\Omega_{T N}, x^{3}, x^{3^{\prime}}\right)$ of the operator $\hat{L}_{S}(\omega)$ at the frequency $\Omega_{T N}$, satisfying the equation

$$
\hat{L}_{S}\left(\Omega_{T N}\right) G_{S}\left(\Omega_{T N}, x^{3}, x^{3^{\prime}}\right)=\delta\left(x^{3}-x^{3^{\prime}}\right) .
$$

Using this expression and Eq. (55) we obtain

$$
\begin{array}{rl}
w_{T N 3}=\frac{1}{2} \oint \oint d & d x^{3} d x^{3^{\prime}} \alpha_{0}\left(x^{3}\right) \alpha_{0}\left(x^{3^{\prime}}\right) \\
& \cdot T_{N}\left(x^{3}\right) T_{N}\left(x^{3^{\prime}}\right) G_{S}\left(\Omega_{T N}, x^{3}, x^{3^{\prime}}\right) .
\end{array}
$$

A more instructive expression for $k_{1 N}^{2}$ can be obtained if the longitudinal WKB-approximation is used. Using Eqs. (65)-(67), after some rearrangement, we obtain:

$$
\begin{aligned}
k_{1 N}^{2}=- & \frac{k_{2}^{2}}{\omega^{2}-\Omega_{T N}^{2}} \\
& \times\left[-\frac{1}{t_{A}} \oint \frac{A}{p} \frac{\partial^{2} p^{-1}}{\partial l^{2}} d l-\frac{\omega}{2 \pi N} \oint \frac{A}{p^{2}} \frac{2}{R} \eta d l\right] .
\end{aligned}
$$

Here $d l=\sqrt{g_{3}} \mathrm{~d} x^{3}$ is an element of the physical length along a field line.

Let us ascertain the sign of the expression between braces. First of all, the first term (with a minus-sign) that is the WKB-expression of the quantity $w_{T N 1}$, is positive, as shown on an earlier occasion in Paper I. (Note that this term is small compared with the second, and it will be neglected in what follows). As far as the quantity $\eta$ is concerned, it can be both larger and smaller than zero. The latter is possible, provided that pressure decreases abruptly with increasing $x^{1}$. Possibly, such a situation is realized during strong substorms. By designating $L_{p}$ a typical scale of decreasing pressure, we obtain the negativity condition for the quantity $\eta$ :

$L_{p}<\frac{R}{2 \gamma}$

(here $\gamma$ is the adiabatic exponent). If this is the case, then $k_{1 N}^{2}>0$ when $x^{1}<x_{T N}^{1}$, i.e., the transparency region in the magnetosphere lies inside the toroidal resonance shell. At quiet geomagnetic epochs, the situation $\eta>0$ seems to us to be more probable, with the transparency region occurring when $x^{1}>x_{T N}^{1}$.

8.2 Behavior of the function $k_{1 N}^{2}\left(x^{1}\right)$ near the magnetosonic resonance surface

In the region near the SMS-resonance, the inequality

$\left|\omega^{2}-\Omega_{S N}^{2}\right| \ll \omega^{2}$ holds. It is evident from Eq. (56) that in this region a compensation of the large value of $k_{1 N}^{2}$ is possible, provided that the function $H_{N}$ has the same order of magnitude. We introduce the substitution:

$\tilde{H}_{N}=\frac{k_{2}^{2}}{k_{1 N}^{2}} H_{N}$.

As follows from the foregoing discussion, the function $\tilde{H}_{N}$ is regular on the SMS-resonance surface. When the condition $k_{1 N}^{2} / k_{2}^{2} \rightarrow \infty$ is satisfied, near this surface the system of Eqs. (55), (56) assumes the form

$$
\begin{aligned}
& \hat{L}_{S}(\omega) \tilde{H}_{N}=\alpha_{0} \Omega_{S N} \frac{k_{2}^{2}}{k_{1 N}^{2}} F_{S N}, \\
& \hat{L}_{T}(\omega) F_{S N}=\alpha_{0} \Omega_{S N} \tilde{H}_{N}
\end{aligned}
$$

$\left(F_{S N}\right.$ designates the function $\left.F_{S N}\left(x^{3}\right)=F_{N}\left(x_{S N}^{1}, x^{3}\right)\right)$. It is evident from Eq. (76) that in the main order, when $\omega=\Omega_{S N}$ and $k_{2}^{2} / k_{1 N}^{2}=\infty$, Eq. (63) holds, i.e. $\tilde{H}_{N}=U_{N}$. In the next order we have

$\tilde{H}_{N}=U_{N}+u_{N},\left.u_{N}\right|_{ \pm}=0$,

where $u_{N}$ is a small correction. By linearizing Eq. (76), we obtain:

$$
\hat{L}_{S}\left(\Omega_{S N}\right) u_{N}+s^{2} \sqrt{g} \frac{\omega^{2}-\Omega_{S N}^{2}}{\omega^{2} v_{S}^{2}} U_{N}=\alpha_{0} \Omega_{S N} \frac{k_{2}^{2}}{k_{1 N}^{2}} F_{S N} .
$$

By multiplying this expression by $U_{N}$ and integrating along a field line, we obtain, using the Hermitian character of the operator $\hat{L}_{S}(\omega)$ and the normalization Eq. (64):

$k_{1 N}^{2}=-k_{2}^{2} \frac{\omega^{2}}{\omega^{2}-\Omega_{S N}^{2}} w_{S N}$,

where it is designated

$w_{S N}=\oint \alpha_{0} F_{S N} U_{N} d x^{3}$

The function $F_{S N}$ will be expressed from Eq. (76) using Green's function $G_{T}\left(\Omega_{S N}, x^{3}, x^{3^{\prime}}\right)$ of the operator $\hat{L}_{T}(\omega)$ satisfying the equation

$\hat{L}_{T}\left(\Omega_{S N}\right) G_{T}\left(\Omega_{S N}, x^{3}, x^{3^{\prime}}\right)=\delta\left(x^{3}-x^{3^{\prime}}\right)$.

We obtain:

$$
\begin{aligned}
w_{S N}= & -\oint \oint d x^{3} d x^{3^{\prime}} \alpha_{0}\left(x^{3}\right) \alpha_{0}\left(x^{3^{\prime}}\right) \\
& \times S_{N}\left(x^{3}\right) S_{N}\left(x^{3^{\prime}}\right) G_{T}\left(\Omega_{T N}, x^{3}, x^{3^{\prime}}\right) .
\end{aligned}
$$

It is easy to make an estimate of $w_{S N}$ in the longitudinal WKB-approximation. We now write the final result:

$k_{1 N}^{2}=-\frac{k_{2}^{2}}{t_{S}} \frac{1}{\omega^{2}-\Omega_{S N}^{2}} \oint \frac{v_{S}}{p^{2}}\left(\frac{2 s}{R A}\right) d l$.

Obviously, the integral on the right-hand side is positive. This means that the second transparency region lies at $x^{1}<x_{S N}^{1}$, i.e., inside the SMS-resonance shell. 


\subsection{Zeroes of the function $k_{1 N}^{2}\left(x^{l}\right)$ : poloidal surfaces}

A knowledge of the behavior of the function $k_{1 N}^{2}\left(x^{1}\right)$ near resonance surfaces is still insufficient for studying the transparency regions of the mode. It is also necessary to ascertain the position of the zeroes of this function, i.e., the points at which the relationship $k_{1 N}^{2}=0$ holds. Magnetic surfaces on which this equality is satisfied will be called the poloidal surfaces. Assuming in Eqs. (55), (56) $k_{1}^{2}\left(x^{1}\right)=0$ we obtain the following system of equations:

$\hat{L}_{S}(\omega) H_{N}=\alpha_{0} \omega F_{N}$,

$\hat{L}_{P}(\omega) F_{N}-\frac{J_{0} \alpha_{1}}{\sqrt{g_{2}}} F_{N}=\alpha_{0} \omega H_{N}$.

It is evident from Eq. (80) that when $\beta=0$ the second equation is brought to the form

$\hat{L}_{P}(\omega) F_{N}=0$.

A coincidence of $\omega$ with the eigenfrequency $\Omega_{P N}\left(x^{1}\right)$ of the operator $\hat{L}_{P}$ (poloidal frequency) gives a coordinate of the poloidal surface in a cold plasma which turns out to be noncoincident with the toroidal surface (for the same $N$ ) because of the difference between corresponding operators caused by the variable character of the quantity $p=\left(g_{2} / g_{1}\right)^{1 / 2}$ along a field line.

If, however, $\beta \neq 0$, and $R^{-1}>0$, then for determining the position of the poloidal surfaces, it is now necessary to solve a system of two differential equations, Eqs. (79), (80). In this case the main factor determining the difference between the toroidal and poloidal surfaces is, as is apparent from this system, the interaction between the Alfvén and SMS-modes. The system of Eqs. (79), (80) is equivalent to one fourth-order equation. To study qualitatively the position of the poloidal surfaces we will use the WKB-approximation in longitudinal coordinate, and limit ourselves to its first order. In addition, we will be unable to "catch" the contribution caused by the difference between the operators $\hat{L}_{T}(\omega)$ and $\hat{L}_{P}(\omega)$, because, as can be demonstrated, this would require using the second order of the WKBapproximation. Incidentally, when $\beta \sim 1$ this contribution is small compared to the contribution due to the mode interaction. In the main order of the WKBapproximation the system of Eqs. (79), (80) is equivalent to an algebraic equation for $\omega$ :

$$
\begin{aligned}
\left(\frac{\omega^{2}}{A^{2}}-k_{\| N}^{2}\right)\left(\frac{\omega^{2}}{v_{S}^{2}}-k_{\| N}^{2}\right) & \\
& -\frac{J_{0} \alpha_{1}}{\sqrt{g_{2}}}\left(\frac{\omega^{2}}{v_{S}^{2}}-k_{\| N}^{2}\right)-\frac{\alpha_{0} \omega^{2}}{s^{2} \sqrt{g_{2}}}=0,
\end{aligned}
$$

where $k_{\| N}$ is the longitudinal wave number determined from the quantization condition

$\oint k_{\| N}\left(x^{3}\right) d x^{3}=2 \pi N$

resulting from the boundary conditions of Eqs. (58), (59). Equation (81) has two roots on $\omega^{2}$. Therefore, there must also be two poloidal surfaces (each of them corresponding to one root $\omega^{2}$ ). Let the coordinates of these surfaces be designated by $x_{P S N}^{1}$, and $x_{P T N}^{1}$, where the letters $S$ and $T$ indicate that one of these surfaces lies nearer to the SMSresonance, and the other lies nearer to the Alfvén resonance (toroidal surface). Formulas resulting by solving Eq. (81) are too unwieldy and virtually unsuitable for further investigation. Therefore, once again, we must resort to the perturbation method, namely, the connection between the hydromagnetic branches will be considered weak, which corresponds to the smallness of the second term in Eq. (81). In the zeroth-order approximation, from Eq. (81) we then have:

$k_{\| N}^{2}=\frac{\omega^{2}}{v_{S}^{2}}$ or $k_{\| N}^{2}=\frac{\omega^{2}}{A^{2}}$.

This means that, with a weak connection between the Alfvén and SMS-branches, the poloidal surfaces must be very close to the resonance surfaces. We now consider the surface which lies nearer to the SMS-resonance. On this surface, the relationship

$k_{\| N}^{2}=\frac{\omega^{2}+\kappa_{S}}{v_{S}^{2}}, \quad\left|\kappa_{S}\right| \ll \omega^{2}$

holds. On substituting this equality into Eq. (81) and using Eq. (82), we obtain

$\omega=\Omega_{P S N} \equiv \Omega_{S N}-\frac{1}{4 \pi N} \oint v_{S}\left(\frac{2 s}{R A}\right)^{2} d l$.

Since the integral on the right-hand side is positive, $\Omega_{P S N}<\Omega_{S N}$. If these functions are both decreasing (which occurs in most of the magnetosphere), hence we obtain $x_{P S N}^{1}<x_{S N}^{1}$.

The other poloidal surface lies nearer to the Alfvén resonance. On this surface

$k_{\| N}^{2}=\frac{\omega^{2}+\kappa_{A}}{A^{2}}, \quad\left|\kappa_{A}\right| \ll \omega^{2}$.

Using the same method as applied in obtaining Eq. (31), we find

$\omega=\Omega_{P T N} \equiv \Omega_{T N}+\frac{1}{4 \pi N} \oint \frac{2}{R} \eta A d l$.

Obviously, all that has been said in regard to the analogous term in Eq. (73) applies for the sign of the integral in Eq. (84): to the case $\eta>0$ there corresponds $\Omega_{P T N}>\Omega_{T N}$ and $x_{P T N}^{1}>x_{T N}^{1}$, and to the case $\eta<0$ there correspond $\Omega_{P T N}<\Omega_{T N}$ and $x_{P T N}^{1}<x_{T N}^{1}$.

It is also easy to ascertain the behavior of the quantity $k_{1 N}^{2}$ near the poloidal surfaces. Without going into details of the mathematics, we give the final expressions:

$k_{1 N}^{2}=-k_{2}^{2} \frac{\left(\omega^{2}-\Omega_{P N}^{2}\right)}{w_{P N}}$,

where

$w_{P N}=-\oint F_{N}\left(x_{P N}^{1}\right) \hat{L}_{T}\left(\Omega_{P N}\right) F_{N}\left(x_{P N}^{1}\right) d x^{3}$. 
The quantity $w_{P N}>0$ if the poloidal surface is nearer to Earth than the resonant surface and $w_{P N}<0$ in the opposite case. It is evident from Eqs. (73), (78), (85) that the transparency region (where $k_{1}^{2}>0$ ) lies only in the interspace bounded by the poloidal surface on one side and by the resonance surface on the other side (Fig. 1).

It follows from Eqs. (83) and (84) that a typical width of the transparency regions is $\Delta_{N} \sim L / N$ (projected onto the equatorial plane). This means that a very large number of transverse wavelengths fit in the transparency region (remember that $m \gg 1$ ); at least this situation is true for the first several $N$-harmonics. For this reason, the WKB-approximation in radial coordinate is obviously applicable. In a cold plasma, the situation is somewhat different: as shown in Paper I, in this case $\Delta_{N} \sim 10^{3}-10^{4} \mathrm{~km}$ at $N=1$ and $\Delta_{N} \sim 100 \mathrm{~km}$ at $N \geq 2$. If at $N=1$ the validity conditions for the transverse WKB-approximation coincide with the condition $m \gg 1$, then, even beginning with the second longitudinal harmonic, they become much more stringent, $m \gg 10$ or even $m \gg 100$, depending on the geomagnetic field model. At the same time, observations show most poloidal pulsations to be $N=2$ and $m \sim 50$ (Anderson, 1994), and that in the cold case (Paper I) the validity of the WKB-approximation is questionable.
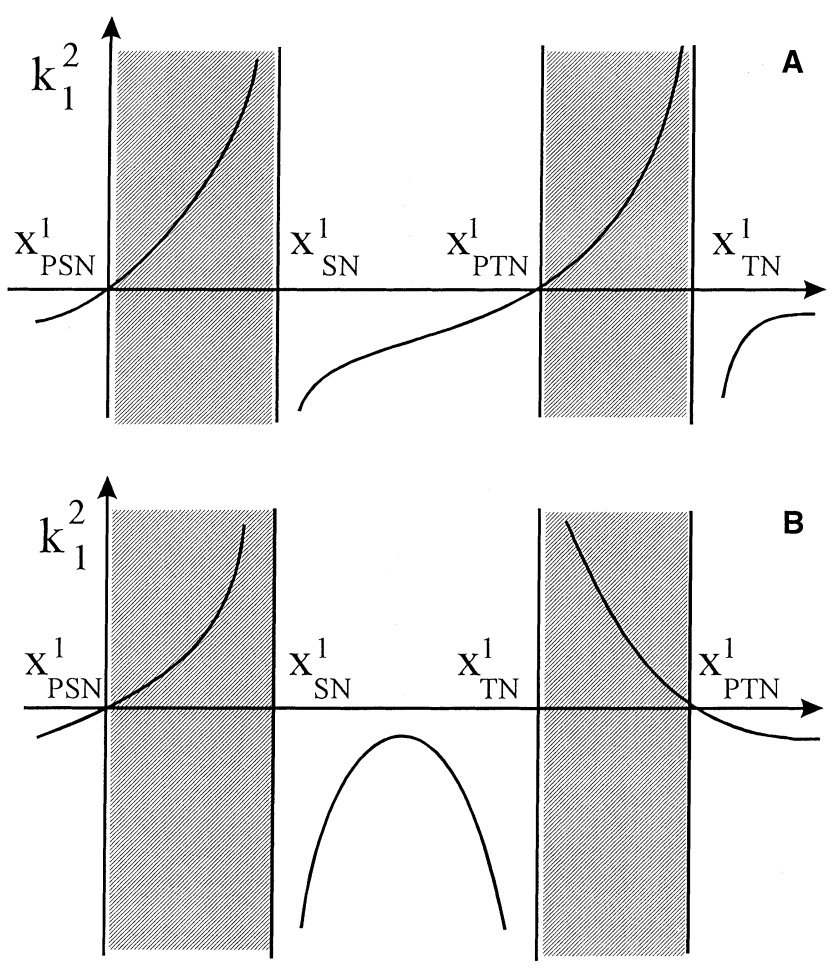

Fig. 1A, B. Schematic representation of the function $k_{1 N}^{2}$ in the cases a normal dispersion $\eta<0$, and $\mathbf{b}$ anomal dispersion $\eta>0$

\section{The group velocity of oscillations}

Thus there is a common situation when the transparency region is bounded on one side (in $x^{1}$ ) by the turning point (poloidal surface) and on the other side by a singular turning point (resonance surface). In this section we consider the question of the propagation of oscillations inside the transparency region. Equations (74), (78), (85) give a dependence of the radial component of the wave vector on the frequency $\omega$. Using these formulas one can calculate the radial component of the wave's group velocity:

$v_{g r}^{1}=\left(\frac{\partial k_{1}}{\partial \omega}\right)^{-1}$.

On differentiating Eq. (73), we obtain the value of the group velocity near the toroidal surface:

$v_{g r}^{1}=\frac{\left(\omega^{2}-\Omega_{T N}^{2}\right)^{2}}{k_{2}^{2} v_{p h}^{1} w_{T N}} \sim\left(\omega^{2}-\Omega_{T N}^{2}\right)^{3 / 2}$,

where $v_{p h}^{1}=\omega / k_{1}$ is the value of the phase velocity. When deriving this relationship, the condition of Eq. (56) is taken into account. Near the SMS-resonance surface

$v_{g r}^{1}=\frac{\left(\omega^{2}-\Omega_{S N}^{2}\right)^{2}}{k_{2}^{2} v_{p h}^{1} w_{S N} \omega^{2}} \sim\left(\omega^{2}-\Omega_{S N}^{2}\right)^{3 / 2}$.

Near poloidal surfaces, from Eq. (85) we obtain:

$v_{g r}^{1}=\frac{w_{P N}}{v_{p h}^{1}} \sim\left|\omega^{2}-\Omega_{P S N}^{2}\right|^{1 / 2}$.

It is evident from Eq. (88) that in the SMS transparency region, the phase and group velocity directions coincide (normal dispersion). In the Alfvén transparency region, as follows from Eq. (87), when $\eta<0$, these directions coincide, while when $\eta>0$ they are opposite (anomalous dispersion). Note that the directions of the phase and group directions coincide if the transparency region is located outside the poloidal shell bounding it (i.e., it lies farther from the Earth than the corresponding poloidal surface), and are oppositely directed if the transparency region lies inside the poloidal shell. Interestingly, the same result was reported by Leonovich and Mazur (1995b) in their investigation on kinetic Alfvén waves in the magnetosphere. Further, it follows from Eqs. (87)-(89) that near the resonance and poloidal surfaces the group velocity goes to zero. However, the typical value of this quantity deep in the transparency regions can be estimated as

$v_{g r}^{1} \sim \frac{A}{m}$

in the "Alfvén" region and

$v_{g r}^{1} \sim \frac{\beta^{2} A}{m}$

in the "magnetosonic" transparency region. The presence in the denominator of a large number of $m$ indicates that the transverse group velocity of wave 
propagation is significantly smaller than the typical Alfvén velocity and the velocity of slow magnetosound.

\section{Structure of hydromagnetic waves near resonance surfaces and in the region of poloidality of the mode}

In this section we derive the transverse solutions for MHD waves near the resonance and poloidal surfaces to examine the details of wave propagation there. Here we shall use a pertubation method similar to the one used in Paper I.

\subsection{Wave structure near the toroidal resonance surface}

Near the toroidal resonance surface, the system of Eqs. (36), (37) can be considerably simplified, because near the resonance surfaces the following condition is satisfied:

$\left|\frac{1}{\sqrt{g_{1}}} \partial_{1} \Phi_{N}\right| \gg\left|\frac{1}{\sqrt{g_{2}}} k_{2} \Phi_{N}\right|$

and, furthermore, near the toroidal surface the wave's frequency $\omega$ differs little from the eigenfrequency $\Omega_{T N}$ of the toroidal operator $\hat{L}_{T}(\omega)$ according to Eq. (71).

In the zero order of perturbation theory when the terms of Eq. (37), proportional to $k_{2}^{2}$, as well as the difference of the frequencies $\omega$ and $\Omega_{T N}$ should be neglected, we get Eq. (61). This means that the function $\Phi$ must be proportional to the eigenfunction $T_{N}$ of the toroidal operator [normalized by the condition of Eq. (62)] up to the factor $V_{T N}$, dependent on $x^{1}$. This factor is determined in the next order of perturbation theory in which the function $\Phi$ is representable as

$\Phi_{N}=V_{T N}\left(x^{1}\right) T_{N}\left(x^{1} \cdot x^{3}\right)+\phi_{N}\left(x^{1}, x^{3}\right)$,

where $\Phi_{N}$ is a small correction, $\left|\phi_{N}\right| \ll\left|\phi_{N}\right|$, caused by the inequality of $\omega^{2}$ and $\Omega_{T N}^{2}$.

Let us make some estimates. A typical value of the derivative of the function $T_{N}$ in radial coordinate is

$\partial_{1} T_{N} \sim \frac{T_{N}}{L}$,

where $L$ is a typical scale of variation of equilibrium parameters of the magnetosphere. However, the scale of variation of the function $\Phi$ must be significantly smaller not only than $L$ but also than $L / m$, in order for Eq. (90) to be satisfied. This condition can be satisfied, provided that the function $V_{T N}\left(x^{1}\right)$ obeys the relationship

$\left|\frac{1}{V_{T N}} \partial_{1} V_{T N}\right| \gg\left|\frac{1}{T_{N}} \partial_{1} T_{N}\right|$.

Boundary conditions for the function $\Phi$ are developed in Sect. 4 Eqs. (42), (43). Based on these equations, we write

$\left.T_{N}\right|_{ \pm}=0,\left.\quad \phi_{N}\right|_{ \pm}=\mp\left(i a V_{T N} \partial_{3} T_{N}\right)_{ \pm}$,

where the + and - signs correspond to the points of intersection of the ionosphere by field lines $\left(x^{3}=x_{ \pm}^{3}\right)$, and $a$ is a small quantity caused by the dissipation of the mode on the ionosphere. We do not include in these conditions the term proportional to the external fieldaligned current in the ionosphere because it can be shown that the inclusion of this term is important only near the poloidal surfaces.

On substituting into Eq. (37) Eq. (91), multiplying the resulting expression by $T_{N}$ and integrating it along a field line, we obtain the following ordinary differential equation:

$\partial_{1}\left[\left(\omega+i \gamma_{N}\right)^{2}-\Omega_{T N}^{2}\right] \partial_{1} V_{T N}-k_{2}^{2} w_{T N} V_{T N}=0$,

where $\gamma_{N}$ is the damping decrement of the mode on the ionosphere determined from the integral

$\oint T_{N} \hat{L}_{T}\left(\Omega_{T N}\right) \partial_{1} \partial_{1} \phi_{N} d x^{3}=2 i \omega \gamma_{N} \partial_{1} \partial_{1} V_{T N}$,

that is nonzero by virtue of the boundary conditions given by Eq. (93). Equations $(62,92)$ were used when deriving Eq. (94).

Near the toroidal surface, one can make use of the linear expansion

$\omega^{2}-\Omega_{T N}^{2}=\omega^{2} \frac{x^{1}-x_{T N}^{1}}{L}$.

We introduce a dimensionless variable

$\xi=\frac{x^{1}-x_{T N}^{1}}{\lambda_{T N}}$,

where

$\lambda_{T N}=\frac{\omega^{2}}{k_{2}^{2}\left|w_{T N}\right| L}$

is a typical radial wavelength near the toroidal surface. Then Eq. (94) may be rewritten as

$\frac{d}{d \xi}\left(\xi+i \epsilon_{T N}\right) \frac{d V_{T N}}{d \xi} \mp V_{T N}=0$,

where it is designated

$\epsilon_{T N}=\frac{2 L \gamma_{N}}{\omega \lambda_{T N}}$

and the - and + signs correspond to the cases $w_{T N}>0$ and $w_{T N}<0$, respectively. As shown in Sect 8, the sign of $w_{T N}$ is opposite to the sign of the quantity $\eta$. When $\eta>0$ the transparency region lies at $x^{1}>x_{T N}^{1}$, and when $\eta<0$ it lies at $x^{1}<x_{T N}^{1}$. According to the boundary condition Eq. (33), in the opacity region the solution must be bounded.

Consider the case $w_{T N}>0$. In this case Eq. (98) reduces to a modified zero-order Bessel equation, the solution of which - bounded in the opacity region - has the form

$V_{T N}=C_{T} \mathbf{K}_{0}(2 \sqrt{\xi})$,

where $C_{T}$ is an arbitrary constant. The asymptotic representation of this solution when $\xi \rightarrow \infty$ (deep in the opacity region) is 
$V_{T N}=C_{T} \frac{\sqrt{\pi}}{2} \xi^{-1 / 4} e^{-2 \sqrt{\xi}}$,

and when $\xi \rightarrow-\infty$ (in the transparency region)

$V_{T N}=C_{T} \frac{\sqrt{\pi}}{2}(-\xi)^{-1 / 4} e^{-2 i \sqrt{-\xi}-i \pi / 4}$.

It is evident from Eq. (100) that the solution in the transparency region has the form of a wave whose phase velocity is directed to the resonance point. When $w_{T N}>0$, the directions of the phase and group velocities coincide (Sect. 9). At the resonance point $\xi=0$ the mode has a logarithmic singularity

$V_{T N} \sim \ln \xi$,

which is regularized through the inclusion of an imaginary addition $i \epsilon_{T N}$.

In the case $w_{T N}<0$ Eq. (98) reduces to the zero-order Bessel equation, the solution of which - bounded in the opacity region - has the form

$V_{T N}=C_{T} \mathbf{H}_{0}^{(1)}(2 \sqrt{\xi})$.

The asymptotic representation of this solution in the opacity region $(\xi \rightarrow-\infty)$ is

$V_{T N}=C_{T} \sqrt{\frac{2}{\pi}}(-\xi)^{-1 / 4} e^{-2 \sqrt{-\xi}}$,

and in the transparency region $(\xi \rightarrow \infty)$ the asymptotic representation is

$V_{T N}=C_{T} \sqrt{\frac{2}{\pi}} \xi^{-1 / 4} e^{2 i \sqrt{\xi}-i \pi / 4}$.

Thus, the phase velocity of the wave is directed from the resonance point at which the mode has a logarithmic singularity [Eq. (101)]. As shown in Sect. 5, when $w_{T N}<0$ the directions of the phase and group velocities are opposite. As is evident, near the toroidal surface the group velocity is always directed to the resonance point. Behind this point, outside the transparency region, the solution is evanescent. This may be treated as the absorption of the wave's energy near Alfvén resonance. The singularity is regularized by taking into account the dissipation of the mode on the ionosphere.

Our obtained solutions are matched with the solution in the WKB-approximation obtained in Sect. 8. Indeed, the asymptotic expansions Eqs. (100) and (103) have the form of those written in the WKB-approximation with the radial wave vector

$k_{1}=\xi^{-1 / 2}$.

In view of the definitions given by Eqs. (96) and (97), this coincides with the expression obtained in the WKBapproximation Eq. (73).

It only remains for us to ascertain the behavior of the observed quantities for the wave near the toroidal surface, i.e., primarily the behavior of the wave's electromagnetic field. The observed wave is a mixture of the Alfvén and magnetosonic wave, but SMS does not make any appreciable contribution to the transverse electromagnetic field of the wave. This is because, according to Eqs. (13)-(15), the electromagnetic field of magnetosound is defined in terms of the function $\Psi$, which, according to Eq. (29), is on the order of magnitude by a factor of $m$ less than the function $\Phi$ that defines the electromagnetic field of the Alfvén wave.

To define the electric field of the Alfvén wave, we will use Eq. (14). We obtain

$E_{1}=-\partial_{1} \Phi_{N} \sim\left(x^{1}-x_{T N}^{1}\right)^{-1}$,

$E_{2}=-i k_{2} \Phi_{N} \sim \ln \left(x^{1}-x_{T N}^{1}\right)$,

i.e., $\hat{E}_{1} \gg \hat{E}_{2}$ ("hats" designate the physical components of the vectors). Using Eq. (15) we find the components of the magnetic field of the Alfvén wave

$B_{1}=-\frac{i c}{\omega} \frac{g_{1}}{\sqrt{g}} i k_{2} \partial_{3} \Phi_{N} \sim \ln \left(x^{1}-x_{T N}^{1}\right)$,
$B_{2}=-\frac{i c}{\omega} \frac{g_{2}}{\sqrt{g}} \partial_{1} \partial_{3} \Phi_{N} \sim\left(x^{1}-x_{T N}^{1}\right)^{-1}$,

i.e., $\hat{B}_{1} \ll \hat{B}_{2}$. As far as the longitudinal component of the magnetic field of SMS is concerned, it follows from Eqs. (15), (35) that it has a logarithmic singularity on the toroidal surface:

$B_{3} \sim \ln \left(x^{1}-x_{T N}^{1}\right)$

(this component is of the order of magnitude $B_{3} \sim m \Phi$ ). Thus, we find that near the Alfvén resonance the following relationships hold:

$\hat{E}_{1} \gg \hat{E}_{2}, \quad \hat{B}_{1} \ll \hat{B}_{2}$.

It behaves typically of azimuthally polarized micropulsations (Anderson, 1994). It is generally believed that these the pulsations have small values of the azimuthal wave number, $m \sim 1$, and that they are excited by the resonance interaction with fast magnetosound arriving from the interplanetary medium or from the outer part of the magnetosphere [see reviews by Anderson (1994), and Potapov and Mazur (1994)]. One can see, however, that there is also an alternative possibility: some of these pulsations have large $m$ and represent transversally small-scale waves "running" to the singular point of the Alfvén transparency region.

\subsection{Wave structure in the region of magnetosonic resonance}

In the region of SMS-resonance the inequality Eq. (90) also holds, but Eq. (71) is substituted for Eq. (75). Therefore, the system of Eqs. (36), (37) acquires the form

$$
\begin{aligned}
& \hat{L}_{S}(\omega) \Theta_{N}=\alpha_{0} \omega \Phi_{N}, \\
& \quad-\partial_{1} \hat{L}_{T}(\omega) \partial_{1} \Phi_{N}=k_{2}^{2} \alpha_{0} \omega \Theta
\end{aligned}
$$

We seek the function $\Theta$ in the form

$$
\Theta=\frac{\omega}{\omega^{2}-\Omega_{S N}^{2}} V_{S N}\left(x^{1}\right)\left[U_{N}\left(x^{1}, x^{3}\right)+u_{N}\right] .
$$


The basis for this is the following. As shown in Sect. 8, in the transverse WKB-approximation $\left(k_{1 N}^{2} \Phi_{N}=\right.$ $\left.-\partial_{1} \partial_{1} \Phi_{N}\right)$ near the SMS-resonance the function $\Theta$ has the same type of singularity as $k_{1 N}^{2}: \Theta \sim k_{1 N}^{2}$. But $k_{1 N}^{2}$ in this region behaves as $k_{1 N}^{2} \sim\left(\omega^{2}-\Omega_{S N}^{2}\right)^{-1}$. Hence one would expect that the function $\left(\omega^{2}-\Omega_{S N}^{2}\right) \Theta\left(x^{1}, x^{3}\right)$ is regular, and this does validate the ease of using Eq. (108). In this expression the function $V_{S N}$ obeys the condition

$\left|\frac{1}{V_{S N}} \partial_{1} V_{S N}\right| \gg\left|\frac{1}{U_{N}} \partial_{1} U_{N}\right|$,

where $U_{N}$ is an eigenfunction of the operator $\hat{L}_{S}(\omega)$, normalized by Eq. (64). Equation (108) involves a small correction $u_{N}$ caused by the inequality $\omega^{2}$ and $\Omega_{S N}^{2}$. The boundary condition for this function allows for the contribution of the ionospheric dissipation Eq. (47):

$u_{N}=\mp\left(i a \partial_{3} U_{N}\right)_{ \pm}$.

Let the function $\Phi_{N}$ near the SMS-resonance be represented as

$\Phi_{N}=V_{S N}\left(x^{1}\right) F_{S N}\left(x^{1}, x^{3}\right)$.

Substituting Eqs. (108) and (110) into Eq. (107) gives in the zero order of perturbation theory Eq.(63). In the next order system Eq. (107) may be written as

$$
\begin{aligned}
V_{S N} \hat{L}_{S}\left(\Omega_{S N}\right) u_{N}+V_{S N} s^{2} \sqrt{g} \frac{\omega^{2}-\Omega_{S N}^{2}}{v_{S}^{2}} U_{N} \\
=\left(\omega^{2}-\Omega_{S N}^{2}\right) \alpha_{0} V_{S N} F_{S N}, \\
\quad-\hat{L}_{T}\left(\Omega_{T N}\right) F_{S N}=\frac{V_{S N}}{V_{S N}^{\prime \prime}} \frac{\omega^{2}}{\omega^{2}-\Omega_{S N}^{2}} k_{2}^{2} \alpha_{0} U_{N}
\end{aligned}
$$

(the prime denotes differentiation with respect to $x^{1}$ ). Herefrom we express $F_{S N}$ :

$$
\begin{aligned}
F_{S N} & =-\frac{V_{S N}}{V_{S N}^{\prime \prime}} \frac{\omega^{2}}{\omega^{2}-\Omega_{S N}^{2}} \frac{k_{2}^{2}}{2} \\
& \times \oint \oint \alpha_{0}\left(x^{3^{\prime}}\right) U_{N}\left(x^{3^{\prime}}\right) G_{T}\left(\Omega_{T N}, x^{3}, x^{3^{\prime}}\right) d x^{3} .
\end{aligned}
$$

The last expression is then substituted into Eq. (111). By multiplying the resulting expression by $U_{N}$ and integrating by parts using the Hermitian character of the operator $\hat{L}_{S}\left(\Omega_{S N}\right)$ and the boundary conditions Eq. (109), we obtain the following equation:

$\frac{\left(\omega+i \gamma_{N}\right)^{2}-\Omega_{S N}^{2}}{\omega^{2}} V_{S N}^{\prime \prime}-k_{2}^{2} w_{S N} V_{S N}=0$.

Near the SMS-resonance, the following expansion is applicable

$\omega^{2}-\Omega_{S N}^{2}=\omega^{2} \frac{x^{1}-x_{S N}^{1}}{L}$.

We introduce the dimensionless variable

$\zeta=\frac{x^{1}-x_{S N}^{1}}{\lambda_{S N}}$,

where
$\lambda_{S N}=\frac{1}{k_{2}^{2} w_{S N} L}$

is a typical scale of the solution near the SMS-resonance surface. Equation (112) is then written as

$\left(\zeta+i \epsilon_{S N}\right) \frac{\partial^{2}}{\partial \zeta^{2}} V_{S N}-V_{S N}=0$,

where

$\epsilon_{S N}=\frac{2 L \gamma_{N}}{\omega^{2} \lambda_{S N}}$

The transparency region of this equation lies at $\zeta>0$. Equation (116) is brought to the first-order Bessel equation. The solution, bounded in the opacity region, is

$V_{S N}=C_{S}(-\zeta)^{1 / 2} \mathbf{K}_{1}(2 \sqrt{\zeta})$

( $C_{S}$ is an arbitrary constant). The asymptotic representation of this solution when $\zeta \rightarrow-\infty$ (in the transparency region):

$V_{S N}=C_{S}(-\zeta)^{1 / 4} \sqrt{\frac{\pi}{4}} e^{-2 i \sqrt{-\zeta}-i \pi / 4}$

and in the opacity region $\zeta \rightarrow \infty$

$V_{S N}=C_{S} \zeta^{1 / 4} \sqrt{\frac{\pi}{4}} e^{-\sqrt{\zeta}}$.

The behavior of this solution at the resonance point is regular and is described by the formula

$V_{S N} \sim 1+\zeta \ln \zeta$.

This means that the function $\Theta_{N}$ has at this point a singularity, as it follows from Eq. (108):

$\Theta_{N} \sim\left(x^{1}-x_{S N}^{1}\right)^{-1}$

According to Eq. (117), the phase velocity of the wave is directed to the resonance point. As shown in Sect. 9, in the SMS transparency region the directions of the phase and group velocity coincide. Thus, it follows from our obtained solution that the wave's energy arrives at the SMS-resonance point while it decreases exponentially in the opacity region. The resonance singularity is regularized by the dissipation of the mode on the ionosphere (by the presence of the nonzero decrement $\gamma_{N}$ ). As is apparent, the situation is fully similar to that occurring near the Alfvén resonance.

As in the case of the region near the toroidal surface, the solution near the SMS-resonance is matched with the WKB-solution obtained in Sect. 8 [see Eq. (77)].

We now turn our attention to the study of the observed quantities. As has already been pointed out, when studying the transverse components of the electromagnetic field, it is sufficient to restrict our consideration to the Alfvén wave. As follows from Eq. (119) and the definitions of Eqs. (14), (15), near the SMSresonance the singularity is present only in the $E_{1}, B_{2}$ components of its electromagnetic field:

$E_{1} \sim \ln \left(x^{1}-x_{S N}^{1}\right), \quad B_{2} \sim \ln \left(x^{1}-x_{S N}^{1}\right)$,

i.e., $\hat{E}_{1} \gg \hat{E}_{2}$, and $\hat{B}_{1} \ll \hat{B}_{2}$. 
We now turn to the longitudinal component of the wave's magnetic field $B_{3}$. As follows from Eqs. (15) and (35), this quantity, proportional to $\Theta$, on the SMSresonance surface has a power-law singularity:

$B_{3} \sim\left(x^{1}-x_{S N}^{1}\right)^{-1}$,

i.e., $\hat{B}_{3} \gg \hat{B}_{1}, \hat{B}_{2}$. Since the plasma pressure perturbation near magnetosonic resonance is totally compensated by magnetic pressure [Eq. (39)], the same behavior also applies for plasma pressure:

$\delta P \sim\left(x^{1}-x_{S N}^{1}\right)^{-1}$,

and the oscillations $B_{3}$ and $\delta P$ must proceed out of phase.

It is a behavior that corresponds to compressional Pc5 pulsations (Anderson, 1994). Many authors, beginning probably with Southwood (1977), suppose that these pulsations are standing magnetosonic waves. Such a conclusion is, we believe, inevitable: the large value of the longitudinal component of the magnetic field of these pulsations suggests that they have a magnetosonic origin, but the fast branch of magnetosound is excluded by the fact that these pulsations have large values of the azimuthal wave number, $m \sim 50$ (as mentioned in the Sect. 3, for fast magnetosound with $m \gg 1$ the magnetosphere is opaque). We must emphasize, however, that, according to our theory, SMS-waves in the magnetosphere are not spatially isolated structures but, rather, oscillations near the singular end of the SMS transparency region.

\subsection{Wave structure near the poloidal surfaces}

The relationship

$\left|\frac{1}{\sqrt{g_{1}}} \partial_{1} \Phi_{N}\right| \ll\left|\frac{1}{\sqrt{g_{2}}} k_{2} \Phi_{N}\right|$

holds in the region of poloidality of the mode, and the wave's frequency $\omega$ differs little from the poloidal one:

$\left|\omega^{2}-\Omega_{P N}^{2}\right| \ll \omega^{2}$

(remember that, according to Sect. 8, there exist two sets of poloidal frequencies corresponding to two families of poloidal surfaces bounding the Alfvén and SMS transparency regions). Near the poloidal surfaces the system of Eqs. (36) and (37) may be written as

$\left(\hat{L}_{0}+\hat{L}_{1}\right)\left(G_{0}+G_{1}\right)=0$,

where it is designated

$\hat{L}_{0}=-\left(\begin{array}{cc}\hat{L}_{P}\left(\Omega_{P N}\right)-\alpha_{1} J_{0} / \sqrt{g_{2}} & \alpha_{0} \Omega_{P N} \\ -\alpha_{0} \Omega_{P N} & \hat{L}_{S}\left(\Omega_{P N}\right)\end{array}\right)$,
$\hat{L}_{1}=\left(\begin{array}{cc}\frac{\sqrt{g}}{g_{2}} \frac{\omega^{2}-\Omega_{P N}^{2}}{A^{2}}-\frac{1}{k_{2}^{2}} \partial_{1} \hat{L}_{T}\left(\Omega_{P N}\right) \partial_{1} & -\alpha_{0}\left(\omega-\Omega_{P N}\right) \\ -\alpha_{0}\left(\omega-\Omega_{P N}\right) & \sqrt{g} \frac{S^{2}}{v_{S}^{2}}\left(\omega^{2}-\Omega_{P N}^{2}\right)\end{array}\right)$,

$$
\begin{aligned}
G_{0} & =\left(\begin{array}{c}
V_{P N}\left(x^{1}\right) F_{P N}\left(x^{1}, x^{3}\right) \\
V_{P N}\left(x^{1}\right) H_{P N}\left(x^{1}, x^{3}\right)
\end{array}\right), \\
G_{1} & =\left(\begin{array}{c}
\Phi_{P N}\left(x^{1}, x^{3}\right) \\
\Theta_{P N}\left(x^{1}, x^{3}\right)
\end{array}\right) .
\end{aligned}
$$

Here the matrix $\hat{L}_{0}$ is Hermitian and the function $V_{P N}\left(x^{1}\right)$ describes the radial structure of the mode and is supposed to be fast varying compared with the functions $F_{P N}\left(x^{1}, x^{3}\right)$ and $H_{P N}\left(x^{1}, x^{3}\right)$. Furthermore, it is assumed that $\left|G_{0}\right| \gg\left|G_{1}\right|$ and $\left|\hat{L}_{0}\right| \gg\left|\hat{L}_{1}\right|$. Boundary conditions for the functions $\phi_{P N}, \theta_{P N}$ are

$\left.\phi_{P N}\right|_{ \pm}=-b_{ \pm},\left.\quad \theta_{P N}\right|_{ \pm}=0$

where $b_{ \pm}$is a term proportional to longitudinal external currents in the ionosphere. These boundary conditions follow from Eqs. (43), (47), and the dissipation on the ionosphere has been omitted since this does not affect substantially the solution near the poloidal surfaces.

In the main order of perturbation theory it is assumed that $\omega=\Omega_{P N}$, and we neglect terms containing differentiation with respect to $x^{1}$. We obtain

$\hat{L}_{0} G_{0 N}=0$.

It is natural to choose such a normalization that the relationship

$$
\begin{array}{r}
\oint d x^{3} \frac{\sqrt{g}}{g_{2}} \frac{\omega^{2}}{A^{2}} F_{P N}^{2}+\oint d x^{3} \frac{s^{2} \sqrt{g}}{v_{S}^{2}} H_{P N}^{2} \\
-\oint d x^{3} \alpha_{0} H_{P N} F_{P N}=1 .
\end{array}
$$

be valid.

In the first order of perturbation theory, from Eq.(126) we obtain

$$
V_{P N} \hat{L}_{1} G_{0 N}+\hat{L}_{0} G_{1 N}=0 \text {. }
$$

We multiply this system from the left by the row $\left(\tilde{F}_{P N}\right.$, $\left.\tilde{H}_{P N}\right)$ and integrate along a field line. In view of Eq. (76) and the Hermitian character of the operators $\hat{L}_{P}(\omega)$ and $\hat{L}_{S}(\omega)$, we obtain the following equation for $V_{P N}$ :

$w_{P N} V_{P N}^{\prime \prime}+k_{2}^{2}\left(\omega^{2}-\Omega_{P N}^{2}\right) V_{P N}=-k_{2}^{2} r_{N}$,

where we introduce the notation

$r_{N}=\left[b \frac{g_{1}}{\sqrt{g}} \partial_{3} \tilde{F}_{P N}\right]_{-}^{+}$.

By comparing these expressions, it follows that the inequality $w_{P N}>0$ occurs in the magnetosonic transparency region and in the Alfvén region when $\eta<0$, while the inequality $w_{P N}<0$ holds in the Alfvén region when $\eta>0$.

Near the poloidal surfaces the following expression is applicable

$\omega^{2}-\Omega_{P N}^{2}=\omega^{2} \frac{x^{1}-x_{P N}^{1}}{L}$.

We introduce the new variable 
$\mu=\frac{x^{1}-x_{P N}^{1}}{\lambda_{P N}}$,

where

$\lambda_{P N}=\left(\frac{L\left|w_{P N}\right|}{\omega^{2} k_{2}^{2}}\right)^{1 / 3}$.

Then Eq. (130) may be rewritten as

$\frac{\partial^{2}}{\partial \mu^{2}} V_{P N} \pm \mu V_{P N}=r_{N}$

Here the upper sign corresponds to the case $w_{P N}>0$, and the lower sign corresponds to the case $w_{P N}<0$.

When $w_{P N}>0$ the opacity region lies at $\mu>0$. The solution, bounded in this region, has the form

$V_{P N}=r_{N}[\mathrm{Gi}(\mu)+\operatorname{Ai}(\mu)]$.

The asymptotic representation of this solution when $\mu \rightarrow-\infty$ is

$V_{P N}=r_{N} \mu^{-1}$

and when $\mu \rightarrow \infty$

$V_{P N}=-r_{N} \frac{\pi^{1 / 2}}{\mu^{1 / 4}} \exp \left(\frac{2}{3} i \mu^{3 / 2}+\frac{i \pi}{4}\right)$,

i.e., the phase velocity is directed away from the poloidal surface. According to Sect. 8, the same direction also applies for the group velocity. The wave's amplitude is proportional to the external longitudinal current which could provide a source of oscillations.

When $w_{P N}<0$, the opacity region lies at $m<0$. The solution in this case is obtained from the solution at $w_{P N}>0$ by the substitution $\mu \rightarrow-\mu$, i.e.,

$V_{P N}=r_{N}[\mathrm{Gi}(-\mu)+\operatorname{Ai}(-\mu)]$.

The asymptotic representation of this solution lies deep in the opacity region $(\mu \rightarrow \infty)$

$V_{P N}=-r_{N} \mu^{-1}$,

and in the transparency region $(\mu \rightarrow-\infty)$

$V_{P N}=-r_{N} \frac{\pi^{1 / 2}}{(-\mu)^{1 / 4}} \exp \left(\frac{2}{3} i(-\mu)^{3 / 2}+\frac{i \pi}{4}\right)$.

In this case the phase velocity is directed to the poloidal surface and, consequently, the group velocity is directed away from it, i.e., the wave's energy travels from the poloidal surface deep into the transparency region, as in the case $w_{P N}>0$.

As far as the observed quantities are concerned, it is clear that near the poloidal surfaces $\hat{E}_{1} \ll \hat{E}_{2}, \hat{B}_{1} \gg \hat{B}_{2}$ the relationships hold. This follows at least from the fact that in the region of poloidality of the mode $\hat{k}_{1} \ll \hat{k}_{2}$. A typical value of the radial wavelength near the poloidal surface

$\lambda_{P N} \sim L m^{-2 / 3}$,

which exceeds significantly the azimuthal wavelength $\lambda_{a} \sim L m^{-1}$. In all likelihood, the experimentally observed poloidal pulsations are just the waves near the poloidal end of the Alfvén and magnetosonic transparency regions.

\section{Conclusions}

The major result of our paper is elucidation of the monochromatic MHD wave structure in the magnetosphere having regard to finite plasma pressure and field line curvature. Whereas the waves are standing on the $x^{3}$ coordinate, they appear to be travelling along the $x^{1}$ coordinate (Fig. 2). There are two transparency regions in the magnetosphere, one of them is adjacent to Alfvén resonance and the other is adjacent to SMS resonance (see Fig. 1). On the other side, on the coordinate $x^{1}$ either of the two transparency regions is bounded by the poloidal surface where the wave is generated. The character of field line oscillations changes from radial to azimuthal as the wave propagates from the poloidal to the toroidal surface. On the SMS resonance the longitudinal component of the wave's magnetic field is peaked too. The wave's transverse dispersion is normal in the SMS transparency region while it can be anomalous as well as normal in the Alfvén transparency region. The special case of this picture is the wave structure that has been studied in the paper Leonovich and Mazur (1993). In our paper, we also investigated the important specific case of the oscillations in a finite but low-pressure plasma $(0<\beta \ll 1)$.

Finally, two additional remarks are necessary. One of them is that we studied only the oscillations which have a fixed frequency and azimuthal wave number [Eq. (9)]. But real oscillations in the circumterrestrial plasma are usually broadband both at $\omega$ and $k_{2}$. It restricts the possibilities of comparing our results with experimental data. However, a more general theory of broadband oscillations inevitably has to be based on results of the theory with $\omega$ and $k_{2}$ being fixed.

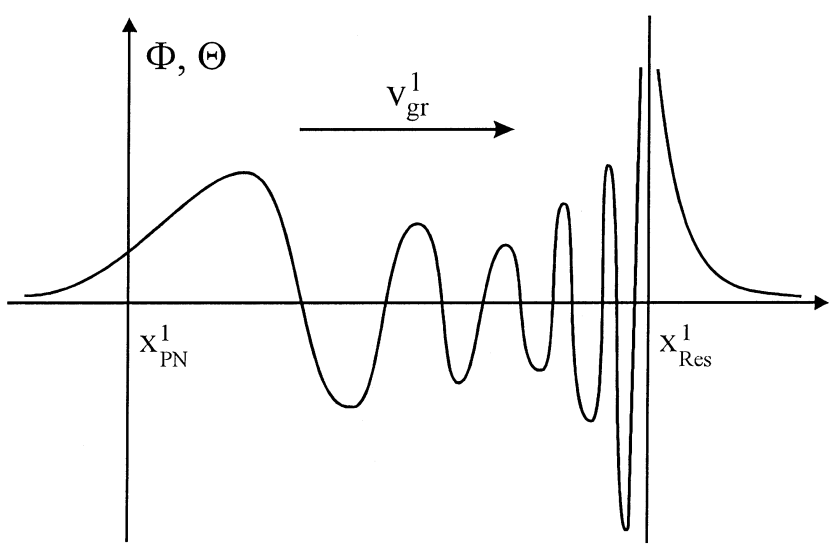

Fig. 2. Schematic representation of the wave's structure in one of the transparency regions lying between the poloidal surface $\left(x_{P N}^{1}\right)$ and the resonance surface $\left(x_{\text {Res }}^{1}\right)$. Shown is the direction of the group velocity: from the poloidal surface to the resonance surface. It should be borne in mind that the mutual arrangement of these surfaces can be different from that shown in the figure: $x_{\text {Res }}^{1}$ can be smaller than $x_{P N}^{1}$ 
The other remark follows from our assumption that the source of the oscillations are ionospheric external currents which could be generated by acoustic movements in the ionosphere. The generation of the variable electromagnetic field in a wide frequency range due to the movements of the neutral component in the ionosphere was studied in works of, for example, Gershman (1974) and Pogoreltzev (1989). The active role of the ionosphere is also discussed in reviews of Lysak (1990) and Pilipenko (1990). At the same time, it is widely believed that the azimuthally small-scale pulsations (e.g., poloidal Pc4, compressional Pc5) are excited by drift and wave-particle instabilities of the magnetospheric plasma. This conception agrees reasonably well with experimental data (Woch et al., 1988; Takahashi et al., 1990). However, instabilities are the amplification mechanism of the waves rather than their source. In order for this mechanism to work, it is necessary to have some "embryos" of the oscillations. The oscillations which are excited by the ionospheric currents can play this role. Thus, we have the following picture: the wave is generated by the source (e.g., external ionospheric currents) and, as it propagates in the magnetosphere, it is amplified due to the instabilities. It should be noted that a theory which describes simultaneously the instabilities, the structure of the waves, and their interaction with the magnetosphere has not yet been created. The longitudinal structure of MHD-waves and their instabilities are treated as a single problem in papers of Ohtani et al. (1989 a, b), Miura et al. (1989), Chen and Hasegama (1991), Walker (1994), Cheng and Qian (1994), but the structure across the magnetic shells was not investigated in these papers.

Acknowledgements. The author is deeply indebted to Dr V. A. Mazur for fruitful discussions without which this work would have been impossible, and for his invaluable advice when writing this paper. Thanks are also due to V. G. Mikhalkovsky for his assistance in preparing the English version of the manuscript. Special thanks to Mikhail.

This work was supported by the International Soros Science Education Program (Grant ISSEP No. A368-F) and by Russian Foundation for Fundamental Research (Grant RFFI No. 96-0216636-a).

Topical Editor K,-H. Glaßmeir thanks I. Mann and A.D.M. Walker for their help in evaluting this paper

\section{Appendix. Derivation of Eqs. (21)-(23)}

The equation of longitudinal oscillations Eq. (21) is the easiest to derive. To do so, it will suffice to consider the longitudinal counter-variance component of Eq. (10) and express in it the displacement in terms of potentials as well as using the relationship of Eq. (8). It is more difficult to derive equations Eqs. (22), (23). We perform, as the starting point, a vector multiplication of Eq. (10) by $\mathbf{B}_{0}$; after some manipulation we obtain in a component-wise representation:

$$
\begin{gathered}
i k_{2} \frac{g_{3}}{\sqrt{g}} i k_{2} E_{1}+\partial_{3} \frac{g_{2}}{\sqrt{g}} \partial_{3} E_{1}+\frac{\sqrt{g}}{g_{1}} \frac{\omega^{2}}{A^{2}} E_{1}-i k_{2} \frac{g_{3}}{\sqrt{g}} \partial_{1} E_{2} \\
=\frac{4 \pi \sqrt{g_{3}}}{B_{0}} \gamma P_{0} K \Theta-\frac{4 \pi \sqrt{g_{3}}}{B_{0}} i k_{2} \frac{i \omega}{c} \xi \cdot \nabla P_{0}, \\
\partial_{1} \frac{g_{3}}{\sqrt{g}} \partial_{1} E_{2}+\partial_{3} \frac{g_{1}}{\sqrt{g}} \partial_{3} E_{2}+\frac{\sqrt{g}}{g_{2}} \frac{\omega^{2}}{A^{2}} E_{2} \\
-\partial_{1} \frac{g_{3}}{\sqrt{g}} i k_{2} E_{1}=\frac{4 \pi i k_{2} \sqrt{g_{3}}}{B_{0}} \partial_{1} \gamma P_{0} \Theta \\
+\frac{4 \pi \sqrt{g_{3}}}{B_{0}} \partial_{1}\left(\frac{i \omega}{c} \xi \cdot \nabla P_{0}\right)-\frac{\sqrt{g_{3}} J_{0}}{\sqrt{g_{2}} B_{0}} \Delta_{\perp} \Psi .
\end{gathered}
$$

This system is representable as

$$
\left(P^{i j}+L^{i j}\right) E_{j}=C^{i}, i=1,2
$$

(we apply the summation over recurring indices here). The operator matrix $P^{i j}$ has the form

$$
\hat{P}=\left(\begin{array}{cc}
\partial_{1} \frac{g_{3}}{\sqrt{g}} \partial_{1} & -\partial_{1} \frac{g_{3}}{\sqrt{g}} i k_{2} \\
i k_{2} \frac{g_{3}}{\sqrt{g}} \partial_{1} & i k_{2} \frac{g_{3}}{\sqrt{g}} i k_{2}
\end{array}\right),
$$

the elements of the operator matrix $L^{i j}$ are

$$
L^{11}=\hat{L}_{T}(\omega), L^{22}=\hat{L}_{P}(\omega), L^{12}=L^{21}=0,
$$

and the components of the vector $C^{i}$ are

$$
\begin{aligned}
C^{1}= & \frac{4 \pi \sqrt{g_{3}}}{B_{0}} k_{2}^{2} \gamma P_{0} \Theta-\frac{4 \pi \sqrt{g_{3}}}{B_{0}} i k_{2} \frac{i \omega}{c} \xi \cdot \nabla P_{0}, \\
C^{2}= & \frac{4 \pi \sqrt{g_{3}}}{B_{0}} i k_{2} \partial_{1} \gamma P_{0} \Theta \\
& +\frac{4 \pi \sqrt{g_{3}}}{B_{0}} \frac{i \omega}{c} \partial_{1}\left(\xi \cdot \nabla P_{0}\right)-\frac{\sqrt{g_{3}} J_{0}}{\sqrt{g_{2}} B_{0}} \Delta_{\perp} \Psi .
\end{aligned}
$$

On substituting into Eq. (A1) instead of $E_{j}$ their expressions in terms of the potentials, we notice that on the left-hand side $P^{i m} \partial_{m} \Phi=0$. By acting on Eq. (A1) with the operator $\partial_{i}$, we obtain Eq. (22), an equation for Alfvén waves (in which it is taken into consideration that through its action on any vector the operator $\partial_{i} P^{\text {im }}$ gives zero). To obtain Eq. (23), we act on Eq. (A1) with the operator

$-\varepsilon^{k j} \partial_{k} \frac{g_{i j}}{\sqrt{g}}$

(where $\varepsilon^{k j}$ is an antisymmetric unit tensor, $\varepsilon^{12}=-\varepsilon^{21}=1, \varepsilon^{11}=\varepsilon^{22}=0$ ). The system of Eqs. (21)(23) is thereby derived.

\section{References}

Anderson, B. J., An overview of spacecraft observations of 10 s to 600s period magnetic pulsations in the Earth's magnetosphere, in Solar wind sources of magnetospheric Ultra-Low-Frequency waves, Geophysical monograph 81, AGU pp. 25-43, 1994.

Chen, L., and A. Hasegama, Kinetic theory of geomagnetic pulsations: 1. internal excitations by energetic particles, J. Geophys. Res., 96, 1503-1512, 1991. 
Cheng, C. Z., and Q. Qian, Theory of ballooning-mirror instabilities for anisotropic pressure plasmas in the magnetosphere, J. Geophys. Res., 99, 11193-11209, 1994.

Cheng, C. Z., T. C. Chang, C. A. Lin, and W. H. Tsai, Magnetohydrodynamic theory of field line resonances in the magnetosphere, J. Geophys. Res., 98, 11339-11347, 1993.

Gershman B. N., The dynamics of ionospheric plas- ma (in Russian), Nauka, Moskow, 1974.

Hargreaves, J. K., The upper atmosphere and solar-terrestrial relations: an introduction to the aerospace enviroment. N.Y. a.o.: VNRC 1979.

Kadomtsev, B. B., Hydromagnetic stability of plasma (in Russian), in Voprosy teorii plazmy, 2, Ed. M.A. Leontovich, Gosatomizdat, Moskow, 132-176, 1963.

Klimushkin, D. Yu., Method of description of the Alfvén and magnetosonic branches of inhomogeneous plasma oscillations, Plasma Phys. Rep., 20, 280-286, 1994.

Klimushkin, D. Yu., A. S. Leonovich, and V. A. Mazur, On the propagation of transversally small-scale standing Alfvén waves in a three-dimensionally inhomogeneous magnetosphere, J. Geophys.Res., 100, 9527-9534, 1995.

Leonovich, A. S., and V. A. Mazur, The spatial structure of poloidal Alfvén oscillations of an axisymmetric magnetosphere, Planet. Space Sci., 38, 1231-1241, 1990.

Leonovich, A. S., and V. A. Mazur, A theory of transverse smallscale standing Alfvén waves in an axially symmetric magnetosphere, Planet. Space Sci., 41, 697-717, 1993.

Leonovich, A. S., and V. A. Mazur, Magnetospheric resonator for transverse-small-scale standing Alfvén waves, Planet. Space Sci., 43, 881-883, 1995a.

Leonovich, A. S., and V. A. Mazur, Linear transformation of the standing Alfvén waves in an axisymmetric magnetosphere, Planet. Space Sci., 43, 885-893, 1995 b.

Lysak, R. L., Electrodynamic coupling of the magnetosphere and ionosphere, Space Sci. Rev., 52, 33-87, 1990.

Miura, A., S. Ohtani, and T. Tamao, Ballooning instability and structure of diamagnetic hydromagnetic waves in a model magnetosphere, J. Geophys. Res., 94, 15231-15242, 1989.

Mond, M., E. Hameiri, and P. N. Hu, Coupling of magnetohydrodynamic waves in inhomogeneous magnetic field configurations, J.Geophys. Res., 95, 89-95, 1990.

Ohtani, S., A. Miura, and T. Tamao, Coupling between Alfvén and slow magnetosonic waves in an inhomogeneous finite-beta plasma: I. coupling equations and physical mechanism, Planet. Space Sci., 37, 567-577, 1989a.

Ohtani, S., A. Miura, and T. Tamao, Coupling between Alfvén and slow magnetosonic waves in an inhomogeneous finite-beta plasma: II. Eigenmode analysis of localized ballooning-interchange instability, Planet. Space Sci., 37, 579-588, 1989 b.

Pilipenko, V. A., ULF waves on the ground and in space, J. Atmos. Terr. Phys., 52, 1193-1209, 1990.
Pogoreltsev, A. I., Electric and magnetic field disturbances caused by the interaction of atmospheric waves with ionospheric plasma (in Russian) Geomagn Aeron 29, 286-292, 1989.

Potapov, A. S., and V. A. Mazur, Pc3 pulsations: from the source in the upstream region to Alfvén resonances in the magnetosphere. Theory and observations, Solar wind sources of magnetospheric ultra-low-frequency waves, Geophysical Monograph 81, AGU, pp. 135-145, 1994.

Saunders, M. A., D. J. Southwood, E. W. Hones, and C. T. Russell, A hydromagnetic vortex seen by ISEE-1 and 2, J. Atmos. Terr. Phys., 43, 927-932, 1981.

Southwood, D. J., Localized compressional hydromagnetic waves in the magnetospheric ring current, Planet. Space Sci., 25, 549$554,1977$.

Southwood, D. J., and M. A. Saunders, Curvature coupling of slow and Alfvén MHD waves in a magnetotail field configuration, Planet. Space Sci., 33, 127-134, 1985.

Takahashi, K., and B. J. Anderson, Distribution of ULF-energy $(f<80 \mathrm{mHz})$ in the inner magnetosphere: a statistical analysis of AMPTE CCE magnetic field data, J. Geophys. Res., 97, 10751-10769, 1992.

Takahashi, K., R. W. McEntire, A. T. Y.Lui, and T. A. Potemra, Ion flux oscillations associated with a radially polarized transverse Pc5 magnetic pulsation, J. Geophys. Res., 95, 37173731, 1990.

Takahashi, K., N. Sato, J. Warnecke, H.E. Spence, and Y. Tonegawa, On the standing wave mode in the giant pulsations, J. Geophys. Res., 97, 10717-10728, 1992.

Taylor, J. P. N., and A. D. M. Walker, Theory of magnetospheric standing hydromagnetic waves with large azimuthal wave number: 2. eigenmodes of the magnetosonic and Alfvén oscillations, J. Geophys. Res., 92, 10046-10052, 1987.

Vetoulis, G., and L. Chen, Global structures of Alfvén-ballooning modes in magnetospheric plasmas, Geophys. Res. Lett., 21, 2091-2094, 1994.

Walker, A. D. M., Theory of magnetospheric standing hydromagnetic waves with large azimuthal wave number: 1 . coupled magnetosonic and Alfvén waves, J. Geophys. Res., 92, 10039$10045,1987$.

Walker, A. D. M., Theory of magnetospheric standing hydromagnetic waves with large azimuthal wave number: 3 particle resonance and instability, J. Geophys. Res., 99, 11105-11112, 1994.

Woch, J., G. Kremser, A. Korth, O. A. Pokhotelov, V. A. Pilipenko, Yu. M. Nezlina, and E. Amata, Curvature-driven drift mirror instability in the magnetosphere, Planet. Space Sci., 36, 383393, 1988.

Zhu, X., and M. G. Kivelson, Compressional ULF waves in the outer magnetosphere: 1.statistical study, J. Geophys. Res., 96, 19451-19467, 1991. 